Research Article: New Research / Disorders of the Nervous System

\title{
Seizure prediction in genetic rat models of absence epilepsy: improved performance through multiple-site cortico-thalamic recordings combined with machine learning
}

https://doi.org/10.1523/ENEURO.0160-21.2021

Cite as: eNeuro 2021; 10.1523/ENEURO.0160-21.2021

Received: 13 April 2021

Revised: 26 October 2021

Accepted: 2 November 2021

This Early Release article has been peer-reviewed and accepted, but has not been through the composition and copyediting processes. The final version may differ slightly in style or formatting and will contain links to any extended data.

Alerts: Sign up at www.eneuro.org/alerts to receive customized email alerts when the fully formatted version of this article is published.

Copyright (@ 2021 Budde et al.

This is an open-access article distributed under the terms of the Creative Commons Attribution 4.0 International license, which permits unrestricted use, distribution and reproduction in any medium provided that the original work is properly attributed. 
1 1. Manuscript title: Seizure prediction in genetic rat models of absence epilepsy: improved

2 performance through multiple-site cortico-thalamic recordings combined with machine

3 learning

\section{Number of Figures: 4}

7. Number of Tables: 3

\section{Number of Multimedia: 0}

\section{Number of words for Abstract: 250}

10. Number of words for Significance Statement: 118

\section{Number of words for Introduction: 678}

12. Number of words for Discussion: 1850 
33 13. Acknowledgements: We would like to thank Thomas Westhoff, Tim Book and Gerard

34 van Oijen for expert IT-support as well as Hubert Bäumer, Petra Berenbrock and Anika 35 Böggemann for animal care. We acknowledge valuable discussion with Prof. Alexander 36 Hramov on brain-computer interfaces and constant valuable support by 'Förderverein 37 Epilepsieforschung an der Universität Münster e.V.'.

14. Conflict of Interest: Authors report no conflict of interest

40 15. Funding sources: This work was supported by a grant from Innovative Medizinische 41 Forschung (IMF) (LÜ121603) to AL, and a DAAD travel grant to VM. 
Abstract

Seizure prediction is the grand challenge of epileptology. Yet, effort was devoted to prediction of focal seizures, while generalized seizures were regarded as stochastic events. Long lasting LFP recordings containing several hundred generalized spike and wave discharges (SWDs), acquired at eight locations in the cortico-thalamic system of wavelet-energy signaling increases in synchronicity. Sensitivity and false alarm rate of prediction were compared between various combinations and wavelet spectra of true- and false positive predictions were fed to a random forest machine learning algorithm to further trace combinations, while predictions based on recordings in layer 4,5 and 6 of the somatosensory-cortex significantly outreached all other combinations in terms of prediction sensitivity. In 24-hours out-of-sample recordings of 9 -GAERS, containing diurnal rem etween false alarms and sensitivity of prediction, as reflected in relatively low F1-score development of closed-loop SWD prediction-prevention systems. Suggestions for a possible translation to human data are outlined.

\section{Significance statement}

4 Seizure prediction was declared the grand challenge of epileptology. While most effort was

5 devoted to the prediction of focal seizures, generalized seizures were regarded as stochastic 6 events. Results of this study demonstrate that above chance prediction of generalized spike 7 and wave discharges (SWDs) is possible in long lasting, pseudoprospective 24 hours 8 recordings of absence epileptic rats, by means of wavelet analysis of LFP traces acquired 9 near the proposed cortical initiation network in S1 and further classification of true and false 10 positive detections by a trained random forest machine learning algorithm. Moreover, as 11 lower SWD prediction performance was achieved by analysis of LFP traces distant to S1, the 12 study provides evidence supporting the cortical focus theory of absence epilepsy. 
Keywords: random forest, artificial neuronal network, absence epilepsy, GAERS, somatosensory cortex, spike and wave discharges

\section{Introduction}

Epilepsy is a neurological disorder characterized by infrequent, short lasting periods of either local or generalized, hypersynchronous brain activity which can be recorded in the electroencephalogram. Depending on the type and nature of these seizures they either go along with a loss of behavioral control in the form of tonic or clonic convulsions and/or with a loss of consciousness. As a majority of patients diagnosed with epilepsy report the uncertainty of when a seizure attack will happen to them as one of the most disabling aspects of the disease, seizure prediction was declared the grand challenge of epileptology (Seizure Gauge Challenge 2017; 2016 Community Survey of Epilepsy Innovation Institute (Ei2) 2016; Kiral-Kornek et al. 2018).

At present, most effort in the development of seizure prediction algorithms has been devoted to the prediction of focal seizures, in which, a local group of abnormally discharging neurons is assumed to gradually recruit a critical mass of neurons during a putative pre-seizure state. Results on seizure prediction performance are quite variable, with multi-variable methods taking measures of synchronization between brain structures into account usually outperforming uni-variable methods (Mormann et al. 2007). Part of this variability can be attributed to methodological shortcomings, and a list of criteria based on which prediction performance should be evaluated was established to guide good scientific practice (Mormann et al. 2007). Criteria include evaluation of prediction performance based on unselected continuous data, in-sample and out-of-sample testing with unseen (pseudo) prospective data, and evaluation with rigorous and solid statistical methods like Monte Carlo surrogate statistics to test prediction performance against chance level prediction (Mormann et al. 2007; Kuhlmann et al. 2018).

More recently developed algorithms evaluated against these criteria, employed machine learning or deep learning approaches, and were found to achieve above chance prediction (Khan et al. 2018; Eberlein et al. 2019; Kiral-Kornek et al. 2018). Both are feature extraction methods that have been proven successful in a number of pattern recognition tasks, like image and speech recognition in medical diagnosis, genomics, translation or robotics (Walter et al. 2019; Ratner 2015; Daily et al. 2017).

Comparatively little effort has been devoted to the prediction of generalized seizures, as they have long been regarded as stochastic events (Lopes Da Silva et al. 2003). In two validated genetic rat model of absence epilepsy (rats of the WAG/Rij strain and Genetic Absence Epilepsy Rats from Strasbourg (GAERS)), characterized by generalized spike and wave discharges (SWDs) and a concomitant decrease in the level of consciousness (Depaulis and 
Charpier 2018; van Luijtelaar and Zobeiri 2014), several studies reported the presence of pre-ictal changes in the corticothalamic system, that might be useful features for SWD prediction (Polack et al. 2007; Sorokin et al. 2016; Pinault et al. 2001; van Luijtelaar et al. 2011; Lüttjohann and van Luijtelaar 2012). A first proof of principle for the predictability of SWDs was provided by Maksimenko et al. (2017). To achieve a measure for synchronization signaling SWD initiation, these authors calculated the product of the wavelet energy assessed in local field potential (LFP) recordings taken at three locations in the corticothalamic system of WAG/Rij rats. While this algorithm already reached a high sensitivity of prediction, it still suffered from a large amount of false alarms, strongly reducing the specificity of prediction.

The current study was designed to improve SWD prediction performance through (i) a systematic variation of the multiple recording sites of SWDs in the cortico-thalamic system and relation to SWD prediction sensitivity and false alarm rate, (ii) a thorough statistical comparison of wavelet spectra corresponding to true positive- and false positive detections, and (iii) training of a machine learning algorithm (random forest) to further differentiate between both types of detections. In line with the criteria of good scientific practice mentioned above, we assessed algorithm performance in long lasting, non-selected, pseudo-prospective 24 hours recordings, taking potential diurnal variations of seizure occurrence into account (Smyk and van Luijtelaar 2020), we incorporated in-sample and out-of-sample recordings (from two different genetic rat models of absence epilepsy, rats of the WAG-Rij strain and GAERS), and we statistically verified the results using surrogate statistics.

\section{Material and Methods}

\subsection{Animals, surgery and acquisition of LFP recordings}

LFP recordings of a total of 22 male WAG/Rij rats and 15 male GAERS, two well validated genetic rat models of absence epilepsy were analyzed. As both strains show several hundred spontaneously occurring SWDs per day (Depaulis and van Luijtelaar 2006), the data are potentially suited for training and evaluation of machine learning algorithms requiring a large amount of training data.

Recordings of 16 WAG/Rij rats were taken from a previously published data set analyzing pre-ictal network interactions in the cortico-thalamic system (Lüttjohann and van Luijtelaar 2012, 2015). In these rats, LFP signals were simultaneously measured in freely moving animals in eight different brain structures within the cortico-thalamic system including the posterior thalamic nucleus (Po), the ventral-postero-medial thalamic nucleus (VPM), caudal and rostral part of reticular thalamic nucleus (cRTN and rRTN), anterior thalamic nucleus 
(ATN) as well as layer IV, V, VI of the somatosensory cortex (S1) (coordinates are specified in Lüttjohann \& van Luijtelaar, 2012). LFP signals were gathered at a constant sample rate of $2048 \mathrm{~Hz}$ and filtered between $1 \mathrm{~Hz}$ high pass (HP) and $100 \mathrm{~Hz}$ (LP) low pass as well as by a $50 \mathrm{~Hz}$ notch filter, over a period of at least 4 hours. A WINDAQ-recording-system was used to digitize EEG signals (DATAQ-Instruments Inc., Akron, OH, USA). Rat movement was registered via a PIR detector (RK2000DPC LuNAR PR Ceiling Mount, Rokonet RISCO Group S.A., Drogenbos, BE). In additional 6 WAG/Rij rats LFP recordings were acquired in layer $\mathrm{Va}, \mathrm{Vb}$ and layer $\mathrm{VI}$ of the secondary motor cortex $(\mathrm{A} / \mathrm{P}+2.7 \mathrm{~mm}, \mathrm{M} / \mathrm{L}+1.2 \mathrm{~mm}, \mathrm{~d}-2.5$, 2.6, $2.8 \mathrm{~mm}$, respectively; coordinates relative to bregma). Coordinates were determined relatively to bregma and according to the stereotactic atlas of Paxinos and Watson (1998)).

LFP recordings of GAERS were acquired in the Münster lab. Animals aged 3 to 9 months, born and raised at the Institute of Physiology I, Westfälische Wilhelms-University Münster. underwent stereotactic surgery under pentobarbital anesthesia (Narcoren, $50 \mathrm{mg} / \mathrm{kg}$; Boehringer Ingelheim Vetmedica $\mathrm{GmbH}$, Ingeheim am Rhein, Germany) for the implantation of recording electrodes (stainless steel, insolated with polyamide, impedance $0.1 \mathrm{M} \Omega$; diameter 0.005 inch; Plastics One, Roanoke, USA) in the deep layer (IV, V and VI) of S1 (A/P: -1.8, M/L: -3.6, d:-2.6, -2.9 -3.2). Reference and a ground electrode were placed on top of the cerebellum. Carprofen $(5 \mathrm{mg} / \mathrm{kg})$ was administered to the rats 30 minutes before as well as 24 and 48 hours after surgery to ensure intra and postoperative analgesia.

Two weeks after surgery animals were placed in a $43 \times 28 \times 42 \mathrm{~cm}$ plexiglas recording box, equipped with bedding material, cage enrichment (Enviro-Dri) and free excess to food and water. Rats were connected to recording leads connected to a swivel commutator allowing LFP recordings in freely moving animals. LFP signals were amplified by an amplifier (TD 90087, Radboud University Nijmegen, Electronic Research Group) filtered between $1 \mathrm{~Hz}$ (HP) and $100 \mathrm{~Hz}$ (LP) as well as by a $50 \mathrm{~Hz}$ notch filter, and digitalized with a constant sample rate of $500 \mathrm{~Hz}$ by WINDAQ-recording-system (DATAQ-Instruments Inc., Akron, OH, USA). In addition, a PIR (Passive Infrared Registration, RK2000DPC LuNAR PR Ceiling Mount, Rokonet RISCO Group S.A., Drogenbos, BE) registered rat movements. GAERS were recorded for a total of 24 hours.

All experimental procedures were carried out according to the guidelines and regulations of the council of the European Union (Directive 2010/63/EU) and were approved by local authorities.

\subsection{Data processing and statistics}

2.2.1. Wavelet-based SWD prediction by the Maksimenko et al (2017) algorithm comparison between combinations of recording sites in the cortico-thalamic system. 
In an attempt to determine the optimal recording sites for SWD prediction and to gain additional insight into network interactions in the cortico-thalamic system in relation to the generation of SWD, we assessed SWD prediction performance in all possible combinations of two and three different recording sites in the cortico-thalamic system (Table I) using the algorithm previously published by Maximenko et al (2017).

For SWD prediction, the Maksimenko et al (2017) algorithm determines in each LFP trace, the mean wavelet energy within a time window of $500 \mathrm{~ms}$ shifting along the complete LFP trace sample by sample. In each trace (i) and at each time step (t), the wavelet energy (W) within the frequency range of $5-10 \mathrm{~Hz}$ corresponding to the precursor $\left(\mathrm{W}_{(5-10 \mathrm{~Hz})}(\mathrm{t})\right)$ is calculated using wavelet transformation with a modified Morlet mother function (Maksimenko et al. 2017; van Luijtelaar et al. 2016). This energy obtained in each trace is multiplied to determine the occurrence of cortico-thalamic synchronization at each moment in time $\left(\mathrm{W}_{(5-10}\right.$ $\left.\mathrm{Hz})(\mathrm{t})=\mathrm{W} 1_{(5-10 \mathrm{~Hz})}(\mathrm{t}) \times \mathrm{W} 2_{(5-10 \mathrm{~Hz})}(\mathrm{t}) \times \mathrm{W} 3_{(5-10 \mathrm{~Hz})}(\mathrm{t})\right)$. Moreover, wavelet energy is calculated and multiplied in each channel for a frequency range of $3-5 \mathrm{~Hz}$ in accordance to the light slow wave sleep $\left(\mathrm{W}_{(3-5 \mathrm{~Hz})}(\mathrm{t})=\mathrm{W} 1_{(3-5 \mathrm{~Hz})}(\mathrm{t}) \times \mathrm{W} 2_{(3-5 \mathrm{~Hz})}(\mathrm{t}) \times \mathrm{W} 3_{(3-5 \mathrm{~Hz})}(\mathrm{t})\right)$ and within a frequency range of $7-20 \mathrm{~Hz}$ representing sleep spindles $\left(\mathrm{W}_{(7-20 \mathrm{~Hz})}(\mathrm{t})=\mathrm{W} 1_{(7-20 \mathrm{~Hz})}(\mathrm{t}) X \mathrm{~W}_{(7-20 \mathrm{~Hz})}(\mathrm{t}) X\right.$ $\left.\mathrm{W}_{(7-20 \mathrm{~Hz})}(\mathrm{t})\right)$ (Figure $\left.4 \mathrm{~A}\right)$.

Decision on whether a SWD precursor is present is based on three criteria:

1. Energy of $\mathrm{W}_{(5-10 \mathrm{~Hz})}(\mathrm{t})$ needs to exceed an individualized specific threshold.

2. Energy of $\mathrm{W}_{(5-10 \mathrm{~Hz})}(\mathrm{t})$ must exceed energy of $\mathrm{W}_{(3-5 \mathrm{~Hz})}(\mathrm{t})$

3. Energy of $W_{(5-10 \mathrm{~Hz})}(t)$ must exceed energy of $W_{(7-20 \mathrm{~Hz})}(t)$

For determination of optimal recording sites for SWD prediction, LFP recordings (duration 4 hours), simultaneously obtained within the cortico-thalamic system in GAERS and WAG/Rij rats, were fed into the wavelet-based SWD prediction algorithm of Maksimenko et al. (2017), testing data from the various recordings sites in all possible combinations (Table I). For WAG/Rij rats, a total number of 57 combinations composed of LFP recordings from three recording sites and 28 combinations, composed of LFP recordings from two recording sites (see Table I), were presented to the algorithm. For GAERS, data from three recording sites in layers IV, $\mathrm{V}$ and $\mathrm{VI}$ of $\mathrm{S} 1$ were used. Each combination of recording sites can be found in Table I; 'C','T','M' globally refers to recording sites in somatosensory cortex (S1), thalamus and secondary motor cortex, respectively.

Since SWD prediction quality depends on the above mentioned individualized threshold, SWD prediction performance of each recording site combination was determined for a total 
of 14 fixed threshold values ranging from 0.1 to 0.75 for all combinations of three recording sites, and a total of 16 fixed threshold values ranging from 0.005 to 0.04 for all combinations composed of two recording sites. Of note, the difference in magnitude in the threshold values for two and three recording sites is attributed to the fact that detection relies on the product of either two or three wavelet energy values (see above). For prediction based on two versus three recording sites, the outer threshold levels (minimum and maximum) correspond to saturated levels of either sensitivity or false alarm rates for all tested combinations.

Detections of the algorithm occurring within a 1s pre-ictal period before SWD onset were regarded as true positives, while detections at interictal timepoints were regarded as false positives. SWD onset was determined according to the criteria outlined by van Luijtelaar \& Coenen (1986), taking the peak of the first spike of twice the amplitude of the background EEG as a reference to mark the onset of the SWD (Figure 1). In case of differences in spike timing between recording sites, notably occurring in the range of milliseconds (Lüttjohann and van Luijtelaar 2012), the peak of the first spike earliest in time was taken as SWD onset.

For each combination of recording sites, and for each of the threshold values, the sensitivity (Sensitivity $=$ number of correctly predicted SWDs / (number of correctly predicted SWDs + number of unpredicted SWDs) $\times 100 \%$ ) of SWD prediction as well as the false alarm rate were determined.

Linear regression analysis (Pearson correlation) was used to determine the degree of interdependence between the sensitivity of prediction and false alarm rate.

Statistical comparison of sensitivity and false alarm rate between different combinations of recording sites were performed using ANOVA with sensitivity or false alarm rate as dependent variable, combination of recording sites as between subject factor 1 , number or recording sites $(2,3)$ as between subject factor 2 , threshold as covariate 1 and false alarm rate or sensitivity as covariate 2 .

To avoid multiple comparison problems all combinations of recording sites were grouped for post-hoc analyses as follows. 1: two intracortical recording sites in S1 (CC), 2: one cortical recording site in $\mathrm{S} 1$ and one thalamic recording site (CT), 3: two intrathalamic recording sites (TT), 4: three intracortical recording sites in S1 (CCC), 5: two cortical recording sites in S1 and one thalamic recording site (CCT), 6: one cortical recording site in $\mathrm{S} 1$ and two thalamic recording sites (CTT), 7: three intrathalamic recording sites (TTT) and 8: three intracortical 
recording sites in the secondary motor cortex (MCCC). Post hoc analyses included: ANOVA with sensitivity or false alarm rate as dependent variable, group of channel combinations (CC, TC, TT, CCC, CCT, CTT, TTT, MCCC) as between subject factor 1 , number of recording sites $(2,3)$ as between subject factor 2 , threshold as covariate 1 and false alarm rate or sensitivity as covariate 2 .

All statistical analyses were performed using IBM SPSS version 25. Data are expressed as the arithmetic mean values \pm standard error of the mean (S.E.M.). Differences were considered statistically significant when $p \leq 0.05\left(^{*}\right), p \leq 0.01\left(^{* *}\right)$ and $p \leq 0.001\left(^{* * *}\right.$.

\subsubsection{Comparison of wavelet spectra corresponding to true positive- and false positive} predictions.

Irrespective of the combination of recording sites, the Maksimenko et al (2017) algorithm results in relatively high false alarm rates. Therefore, we determined pre-existing differences in spectra corresponding to either true positive- or false positive predictions. Wavelet spectra of all true positive detections, and a total number of 50 randomly selected false positive detections, as identified by the algorithm of Maksimenko et al. (2017), were calculated from LFP traces acquired in the deep layer (IV, V and VI) of S1 in GAERS and WAG/Rij rats (Figure 3). Timepoint zero indicates the timepoint of precursor detection at the end of a 500 ms analysis window (ranging from -0.5 to 0 ), in which either the true positive precursor or the false positive was detected.

Average wavelet energy within different frequency bands was statistically compared between true and false detections using repeated measures ANOVA with average wavelet energy as dependent variable, type of detection (true positive, false positive) as within subjects factor 1 , frequency band $\left(\mathrm{W}_{(5-10 \mathrm{~Hz}),} \mathrm{W}_{(3-5 \mathrm{~Hz})}\right.$ and $\left.\mathrm{W}_{(7-20 \mathrm{~Hz})}\right)$ as within subjects factor 2 and rat strain (GAERS, WAG/Rij rats) as between subject factor.

\subsubsection{Random forest machine learning algorithm for differentiation between true positive -} and false positive predictions

In an attempt to further differentiate between true and false positive predictions, we trained a random forest machine learning algorithm. The wavelet energy extracted for true and false detections was fed into a random forest (Birjandtalab et al. 2017) consisting of a total of 1000 decision trees (Figure 4A). Different numbers of trees were experimentally varied to investigate the effect of forest size on classification performance (Figure 4E). For each true and false positive prediction produced by the Maksimenko et al (2017) algorithm, 9 wavelet 
energy values corresponding to the values assessed in the three frequency bands $\left(\mathrm{W}_{(5-10 \mathrm{~Hz}) \text {, }}\right.$ $W_{(3-5 \mathrm{~Hz})}$ and $\left.W_{(7-20 \mathrm{~Hz})}\right)$ at three different recording sites, were presented to the algorithm to extract features for classification (Figure 4A). Majority voting of the different trees in the random forest yielded final classification (Figure 4A).

Training of the random forest was performed with spectra obtained in $70 \%$ of all recorded data in $6 \mathrm{WAG} / \mathrm{Rij}$ rats and 6 GAERS, and classification performance was evaluated on the remaining $30 \%$ of unseen data of the same rats (i.e. in-sample testing). As epileptic seizures or pre-ictal events are underrepresented compared to the vast number of inter-ictal fragments or false positive predictions, a random undersampling approach was taken in a first step in order to create a balanced training set and thereby ensure balanced learning (Kubat et al. 1997). All true positive detections were fed into the algorithm, matched by an equal number of randomly selected false positive detections. In this way a total of 100 random forest were trained. Of note, each random forest was fed with a different set of false positive detections. Obtained results correspond to the performance of a single trained random forest, which was found to reach an average performance of these 100 trained trees.

In order to allow an unbiased comparison of classification performance of the random forest between different combinations of recording sites, we adjusted the detection threshold of the Maksimenko et al algorithm (2017) for each combination to reach a $60 \%$ sensitivity of SWD prediction for the extraction of the time points and wavelet features for training and evaluation of classification.

To assess the classification performance of the random forest the balanced accuracy of classification was calculated as (sensitivity of classification + specificity of classification) / 2), with specificity $=$ (number of false positives predicted as false positives / (number of false positives predicted as false positives + number of false positives predicted as true positives)) * $100 \%$ and sensitivity $=$ (number of true positives predicted as true positives / (number of true positives predicted as true positives + number of true positives predicted as false positives $)){ }^{*} 100 \%$. Moreover an F1-score defined as F1 $=2{ }^{*}$ ((precision * sensitivity) / $($ precision + sensitivity $)) * 100 \%$ was calculated, where precision equals (number of true positives predicted as true positives / (number of true positives predicted as true positives + number of false positives predicted as true positives)) * $100 \%$.

Classification performance of the random forest was compared with ANOVA between the different groups of recording sites in WAG/Rij rats: 1. Recordings in layers $\mathrm{V}$ and $\mathrm{VI}$ of $\mathrm{S} 1$, referred to as "CC" ( $n=145)$; 2. Recordings in layers IV, V and VI of S1, referred to as "CCC" ( $n=161)$; 3. Recordings in layers IV, VI of $S 1$ and VPM, referred to as "CCT" $(n=161) ; 4$. 
Recordings in layer VI of S1, VPM and RTN, referred to as "CTT" ( $n=161) ; 5$. Recordings in VPM, cRTN and Po, referred to as "TTT" $(n=145), 6$. recordings in layer $\mathrm{Va}, \mathrm{Vb}$ and VI of secondary motor cortex, referred to as "MCCC" $(n=161)$. In addition, classification performance was assessed in recordings from layers IV, V and VI of S1 in GAERS, referred to as "GCCC" ( $n=145, n=161, n=1844)$ and compared to results achieved in WAG/Rij rats using ANOVA. Furthermore, classification performance of each group was evaluated against chance level using surrogate statistics (see below).

\subsubsection{Probing the random forest machine learning algorithm for maximal SWD prediction} performance

Next, the random forest machine learning combined with the Maximenko et al. (2017) algorithm were probed for maximal prediction performance of SWD. Wavelet features for true and false predictions were extracted in LFP recordings obtained in the deep layers (IV, $\mathrm{V}$ and $\mathrm{VI}$ ) of S1 of 6 GAERS at a threshold value reaching a $90 \%$ sensitivity for SWD prediction, and were used for training and in-sample testing as described above. Moreover, performance of random-forests trained in this approach were assessed in unseen 24 hours recordings from a separate group of 9 GAERS rats (out-of-sample testing).

For in-sample testing and out-of sample testing, performance was statistically evaluated against chance level prediction using surrogate statistics. To this end, training data of true and false detections were randomly assigned to the two classes (total of 1000 randomizations), and for each randomization the average balanced accuracy achieved in the unseen data was determined and displayed in a histogram. In case the achieved balanced accuracy computed for the random forest trained with the real (i.e. non-randomized) training data was positioned above the 95th quantile of the histogram, algorithm performance was regarded as significant above chance level.

Lastly, as classification performance of the random forest was found to be reduced in the out-of-sample testing, likely resulting from an insufficient amount of false positive predictions presented to the algorithm during training, a separate set of random forests $(n=100)$ was trained in a (moderate) oversampling approach. A multiple (4) of all true positive predictions and a matched number of randomly selected false positive predictions, derived in LFP recordings of the deep layer (IV, V and VI) of S1 in 6 GAERS at a threshold value of $90 \%$, were used to train the random forests. Determination of an appropriate oversampling factor was performed by comparison of classification performances achieved at different oversampling factors, ranging between 2 to 7 . Higher rates of oversampling were omitted to 
avoid overtraining. As for the under-sampling approach, classification performance was assessed in unseen 24 hours recordings from a separate group of 9 GAERS rats (out-ofsample testing) and tested against chance level using surrogate statistics (see above).

Performance presented in the results corresponds to the performance of a single trained random forest, reaching an average performance of these 100 trained trees.

\subsection{Histology}

At the end of the recordings, a direct current $(9 \mathrm{~V}, 25 \mu \mathrm{A}, 2 \mathrm{~s}$ duration) was pathed though each electrode to create an electrolytic lesion at the location of the tip of the electrode. Animals were killed with an intraperitoneal injection of pentobarbital (Narcoren, $150 \mathrm{mg} / \mathrm{kg}$; Merial $\mathrm{GmbH}$, Münster, Germany). The brain was quickly removed and placed in a $4 \%$ paraformaldehyde (PFA) solution for at least $24 \mathrm{~h}$. Brains were fixated in a $30 \%$ sucrose solution and cut into $60 \mu \mathrm{m}$ slices with the aid of a microtome. Slices were mounted on microscope slides, stained with cresyl violet, and inspected under a light-microscope (dnt, DigiMicro Profi) for identification of the microlesions. Recording sites were extrapolated from the center of the lesion relative to cortical depth and neighboring cortical layers. Only recordings from verified recording positions were included in the analysis.

\subsection{Code Accessibility}

The random forest algorithm was programmed in Python and requires previous installation of Python for execution. The code of the random forest algorithm is available as Extended Data.

\section{Results}

\subsection{Electrophysiological characteristics of SWDs in GAERS and WAG/Rij rats} Exemplary LFP recordings of GAERS and WAG/Rij rats are displayed in Figure 1. LFP signals of GAERS, recorded for 24 hours, displayed frequent (average of 17 per hour) SWDs of 10 to 30 seconds duration at a main frequency of $5-7 \mathrm{~Hz}$. Occurrence of SWDs showed the well documented diurnal variation with highest rates of occurrence at the beginning of the dark phase and lowest rates of occurrence at beginning of the light phase (Smyk and van Luijtelaar 2020). LFP signals in WAG/Rij rats were acquired during four hours of the dark phase. WAG/Rij rats showed on average 10 SWDs per hour, with a mean duration of $7 \mathrm{~s}$ and a slightly higher internal frequency of $8-10 \mathrm{~Hz}$. Spikes in thalamus typically possessed a smaller amplitude (500 vs $700 \mu \mathrm{V}$ ) and broader form, with a reversed polarity as compared to those in cortex. All differences of SWD morphology between strains (i.e. different internal 
frequency) and recording sites (i.e. amplitude, polarity and sharpness of spike) are in accordance with previously published data (Sitnikova and van Luijtelaar 2007; Lüttjohann and van Luijtelaar 2012; Akman et al. 2010).

- place figure 1 about here -

- place table I about here -

\subsection{Influence of cortico-thalamic recording sites on SWD prediction performance}

In a first set of experiments, we sought to identify the influence of LFP recording sites on SWD prediction performance. LFP recordings were simultaneously obtained at multiple sites in the cortico-thalamic system of WAG/Rij rats, specifically in the deep layers (IV, V and VI) of the somatosensory cortex (S1), secondary motor cortex, and thalamic nuclei VPM, PO, ATN, rostral and caudal RTN.

Recordings from either two or three sites in all possible combinations (yielding a total of 85 combinations) were fed into the wavelet-based algorithm (Maksimenko et al. 2017). Sensitivity and false alarm rate of the algorithm were compared in these 85 combinations (Table I). For post-hoc analysis combinations were grouped as either 'CC' (two intracortical recording sites in S1), 'CT' (one cortical recording site in S1 and one thalamic recording site), 'TT' (two intrathalamic recording sites, 'CCC' (three intracortical recording sites in S1), 'CCT' (two cortical recording sites in S1 and one thalamic recording site), 'CTT' (one cortical recording site in S1 and two thalamic recording sites), 'TTT' (three intrathalamic recording sites) or 'MCCC' (three intracortical recording sites in the secondary motor cortex), respectively. Moreover, SWD prediction performance of each combination of recording sites was determined at multiple threshold values employed for precursor detection. As ANOVA revealed a significant influence of threshold on both sensitivity of prediction $\left(F(1,10980)=3995, p<0.001, R^{2}=0.26\right)$ (the higher the threshold, the lower the sensitivity) and false alarm rate $\left(F(1,10980)=10.7, p<0.05, R^{2}=0.1\right)$ (the higher the threshold, the lower the false alarm rate), threshold was taken as a covariate factor into statistical analysis in order to allow comparison of prediction performance between different combinations of recording sites irrespective of any possible threshold effects.

ANOVA revealed significant differences in both the achieved sensitivity of prediction as well as the produced false alarm rate between the different combinations of recording sites $\left(F_{\text {senitivity }}(84,10980)=13.47, p<0.001, R^{2}=0.37 ; F_{n F P}(84,10980)=2.47, p<0.001, R^{2}=0.1\right)$ (Figure 2, Table I). 
373

374

375

376

377

On average, predictions based on three recording sites reached significantly higher sensitivities (Figure 2A, Table I) and lower false alarm rates (Figure 2C, Table I) as compared to predictions based on two recording sites $\left(F_{\text {senitivity }}(1,10980)=935.7, p<0.001\right.$, $\left.R^{2}=0.07 ; F_{n F P}(1,10980)=116.3, p<0.001, R^{2}=0.02\right)$.

Regarding the false alarm rate (Figure 2C, Table I) predictions based on three intracortical recordings in S1 (CCC) and predictions based on cortico-thalamic recording sites (CCT and CTT) showed a significantly smaller number of false alarms compared to predictions based on three intrathalamic recordings (TTT) (all $p<0.001$ ) (average false alarm rate of CCC $=$ $85.2 \pm 10.6, \mathrm{CTT}=94.7 \pm 3.0, \mathrm{CCT}=70.6 \pm 3.5$ and $\mathrm{TTT}=110.2 \pm 5.4)$. Predictions based on three intracortical recordings acquired in the secondary motor cortex (MCCC), on the other hand, resulted in significantly more false alarms (average false alarm rate MCCC $=129.8 \pm$ 17.9) as compared to predictions based on CCC, CCT and CTT combinations (all p<0.05). Highest false alarm rates with an average of $221.1 \pm 6.2$ were found for predictions based on two intracortical recordings acquired in $\mathrm{S} 1$ (all $p<0.001$ ).

Regarding the sensitivity of SWD prediction, predictions based on recordings in layer IV, V, and VI of $\mathrm{S} 1$ significantly outreached all other combinations with an average sensitivity of $61.7 \pm 1.5 \%$ (all $p<0.001$ ) (Figure $2 \mathrm{~A}$, Table I).

Among the remaining combinations with three recording sites, MCCC, TTT and CTT showed significantly lower sensitivities compared to predictions based on two recording sites in S1 combined with one thalamic site (CCT) (all $p<0.001$ ) (Figure 2A, B, Table I). Lowest sensitivity was reached for predictions on two thalamic recordings (average sensitivity $\mathrm{TT}=$ $13.7 \pm 0.8 \%$ ), while predictions based on two cortical recording sites in S1 reached a medium sensitivity of $33.0 \pm 0.9 \%$ (Figure $2 \mathrm{~A}, \mathrm{~B}$, Table I).

To estimate the degree of interdependence between achieved sensitivity of SWD prediction and resulting false alarm rate regression analysis was performed. Analysis revealed a significant negative correlation between both indicators of SWD prediction performance $(r=$ 0.716; $p<0.001$ ) (Figure $2 E$ ), indicating that a higher SWD prediction sensitivity, achieved for a given combination of recording sites, does not occur at the trade-off of a high false alarm rate. The same clusters as described above could be identified in the regression pattern including higher false alarm rates and lower sensitivities for predictions on two recording sides within the cortico-thalamic system, highest false alarm rate and medium sensitivity for predictions based of two intracortical recordings in $\mathrm{S} 1$, medium sensitivity and medium false alarm rate for predictions based on three intracortical recordings in $\mathrm{M} 2$ and highest sensitivity with a low false alarm rate for prediction based on three intracortical recordings in S1 (Figure 
2). Of note, irrespective of recording site combination, algorithm performance remained at a low level including only moderate sensitivities of SWD prediction and high false alarm rates.

\subsection{Out-of-sample testing: Comparison between rat strains}

Both, GAERS and WAG/Rij rats are well validated genetic rat models of absence epilepsy sharing genetic, physiological and behavioral characteristics (Depaulis and van Luijtelaar 2006), although slight, but significant differences in electrophysiological parameters of SWDs have been reported (Akman et al. 2010). Therefore, we evaluated the prediction performance of the Maksimenko et al. (2017) algorithm also in GAERS. Prediction performance was assessed in 4 hours lasting LFP recordings, obtained in layers IV, V and VI of S1 in GAERS and WAG/Rij rats, and, was compared between the two strains. Significant differences between rat strains were revealed for the produced false alarm rate, with significantly more false alarms in WAG/Rij rats compared to GAERS ( $p<0.001$ ) (Figure 3B). On the other hand, no significant differences were seen between GAERS and WAG/Rij rats for the sensitivity of prediction $(p>0.05)$ (Figure $3 A)$.

\subsection{Comparison of true and false positive detections}

Irrespective of the combination of recording sites, the Maksimenko et al (2017) algorithm resulted in relatively high false alarm rates. Therefore, we determined pre-existing differences in spectra corresponding to either true positive- or false positive predictions in a next experimental step.

Figure $3 \mathrm{D}$ and $\mathrm{E}$ depict exemplary spectrograms of true and false positive SWD predictions, respectively. Time point -0.5 to 0 features the analysis window (window size $500 \mathrm{~ms}$ ) in which either the true positive precursor or the false positive was detected. The onset of the SWD is depicted at time point 0.4 seconds on the $x$-axis (Figure $3 C, D$ ). At this point a strong increase in the product of the wavelet energy can be noted in the main frequency band of the SWD (i.e. 5-10 Hz). On average, precursor activity around 900 to $300 \mathrm{~ms}$ before SWD onset.

- place figure 3 about here -

Next, the product of wavelet energy, assessed in the frequency bands $\mathrm{W}_{(5-10 \mathrm{~Hz})}, \mathrm{W}_{(3-5 \mathrm{~Hz})}$ and $W_{(7-20 \mathrm{~Hz})}$ (Maksimenko et al. 2017), was statistically compared between true and false positives across the two rat strains. Data revealed significant differences between true and false positives in the frequency bands $W_{(5-10 \mathrm{~Hz})}$ and $W_{(3-5) \mathrm{Hz})}$. False positives possessed a higher wavelet-energy product as compared to true-positives (all $p<0.05$ ). For both frequency 
bands, this difference was significantly more pronounced in GAERS compared to WAG/Rij rats $\left(F(2,28)=7.3, p<0.05, R^{2}=0.3\right)$ (Figure $\left.3 F, G\right)$.

\subsection{A random forest machine learning algorithm for improvement of SWD prediction}

Since significant differences in the wavelet spectra of true and false positives were revealed, a random forest machine learning algorithm was trained to differentiate between true positive and false positive detections. In a first step, a random undersampling approach was used to create a training data set. Here, true positives detected in $70 \%$ of recordings from 6 WAG/Rij or 6 GAERS rats and an equal amount of randomly selected false positives derived from $70 \%$ of recordings in the same rats were used as training data. For in-sample performance evaluation, the algorithm was confronted with the remaining $30 \%$ of unseen data (see methods section for more details). As in the paragraphs above, classification performance of the random forest was compared between different combinations of recording sites in WAG/Rij rats and between rat strains (Figure 4).

- place figure 4 about here -

In WAG/Rij rats, classification performance of the random forest was significantly above chance level for all combinations of recording sites (average balanced accuracy $\mathrm{CCC}=$ $71.5 \%, \mathrm{CCT}=66,7 \%, \mathrm{CTT}=63,2 \%, \mathrm{CC}=62,5 \%$ ) (all $\mathrm{p}<0.05$ ) except for spectra derived from three intrathalamic recording sites (average balanced accuracy TTT $=56,2 \%)(p>0.05)$ and spectra derived from recordings in layer $\mathrm{Va}, \mathrm{Vb}$ and $\mathrm{VI}$ of the secondary motor cortex (average balanced accuracy MCCC $=49,9 \%)(p>0.05)$ (Figure 4B). Highest classification accuracies were derived from three intracortical recordings acquired in $\mathrm{S} 1$, as was seen using the Maksimenko algorithm above (all $p<0.05$ ). Classification accuracies for spectra derived from three intracortical recordings in S1 from GAERS were significantly higher (balanced accuracy GCCC1841 $=78,8 \%$ ) compared to those in WAG/Rij rats (balanced accuracy GCCC1841 $=78,8 \%$ vs. balanced accuracy CCC $=71,5 \%$, p<0.05). Of note, this strain difference could not be attributed to the difference in the amount of training samples (i.e. 1841 derived from $70 \%$ of the six 24 hours recordings of GAERS vs 161 derived from $70 \%$ of the six 4 hours recordings of WAG/Rij rats), as a reduction of the training data in GAERS still resulted in higher classification accuracies than in WAG/Rij rats (balanced GCCC161 $=73.6 \%$ vs balanced accuracy CCC $=71,5 \%, p<0.05$ ) (Figure 4B).

In order to evaluate if classification accuracy of the random forest depends on the level of sensitivity achieved by the Maksimenko algorithm, classification performance in GAERS and WAG/Rij rats achieved at sensitivities of $60 \%$ and $90 \%$ were compared for spectra derived in recordings of layer IV, V and VI in S1. In both strains, a small but significant reduction in 
classification accuracy was noted for spectra derived at a $90 \%$ sensitivity as compared to spectra derived at a $60 \%$ sensitivity (balanced accuracy CCC $=71.5 \%$ vs CCC $90 \%=63.3 \%$ p $<0.001 ;$ GCCC1841 $=78.8 \%$ vs $\operatorname{GCCC} 90 \%=73.1 \% p<0,001)$. Of note, classification accuracies for spectra derived at a sensitivity of $90 \%$ significantly exceeded chance level classification as indicated by surrogate statistics (both $\mathrm{p}<0.01$ ) (Figure 4B). Moreover, accuracies gradually increased towards a maximum at around 16 trees (Figure 4E).

For out-of-sample evaluation, the random forest trained on spectra derived from three intracortical recordings in S1 of GAERS at a sensitivity of $90 \%$ was confronted to spectra derived from 24 hours recordings in a separate group of GAERS $(n=9)$

Table II depicts the achieved balanced accuracies of each rat as well as the average confusion matrix, specifying the relative percentage of true positives that had been classified as such (lower right corner), true positives that had been incorrectly classified as false positives (lower left corner), false positives correctly classified as such (upper left corner), and false positives incorrectly classified as true positives (upper right corner). Classification performance drastically dropped and above chance classification tested by permutation statistics was only achieved in a single rat (i.e. rat 5 , balanced accuracy $59,62 \%, p<0.05$ ).

- place Table II about here -

As the low performance of the random forest in the out-of-sample evaluation might be attributed to random undersampling (i.e. the algorithm was trained with a training set which does not adequately represent the full spectrum/variance of the false positive spectra), we next evaluated the performance of an random forest, which was trained in a (moderate) oversampling approach. In this approach the random forest was trained with four times all true positive detections and a matched number of randomly selected false positive detections, derived in three intracortical recordings in S1 of GAERS at a sensitivity of $90 \%$ (see methods for details). Again, for out-of-sample evaluation, the trained random forest was confronted to spectra derived from 24 hours recordings in a separate group of nine GAERS. Table III depicts the achieved balanced accuracies of each individual rat as well as the average confusion matrix.

- place Table III about here -

Taking this (moderate) oversampling approach, the achieved balanced accuracies of the random forest significantly increased $\left(F(1,8)=26.8, p<0.001, R^{2}=0.7\right)$, and above chance classification could be achieved in all subjects except one (permutation statistics, all but one $\mathrm{p}<0.05$ ) (Table III).

Classification of the random forest trained with the (moderate) oversampling approach resulted in a strong reduction in the false alarm rate. While the Maximenko et al (2017) 
algorithm alone produced an average number of 9388 false alarms within the 24 hours, sorting of the random forest reduced the false alarm rate by $71,4 \pm 2.6 \%$. Reduction of the false alarm rate, however, occurred at some tradeoff between false alarm rate and sensitivity. Here, Maksimenko et al (2017) on average correctly predicted 368 out of 409 SWD, while 40 SWD were not detected (corresponding to a sensitivity of $90 \%$ ). Following sorting by the random forest, an average of 200 out of 409 SWD were correctly predicted (corresponding to a sensitivity of $49 \%$ ). It has to be mentioned, however, that rather large inter-individual differences occurred in prediction performance using the combined "Maksimenko et al + random forest" algorithm. Highest performance was seen in a rat in which 349 out of 520 SWD were correctly predicted (corresponding to a sensitivity of $67 \%$ ).

\section{Discussion}

The current study was designed to improve the prediction of SWDs, a type of generalized seizures seen in several forms of absence epilepsy (Panayiotopoulos et al. 1992). While these types of seizures have long been regarded as stochastic events (Lopes Da Silva et al. 2003), a recent study by Maksimenko et al. (2017) aimed at prediction of SWDs through the use of a dedicated algorithm, which calculates the product of the wavelet energy in LFP recordings taken at three locations in the cortico-thalamic system of absence epileptic rats. A drawback was that this algorithm suffered from a large amount of false positive detections. Therefore, the current study was designed to improve prediction performance, as quantified by sensitivity, specificity and balanced accuracy of prediction. The rational was to systematically vary the sites of simultaneous recordings in the cortico-thalamic system, including somatosensory and motor cortices, rostral and caudal RTN, specific (VPM) and higher order thalamic nuclei (PO, ATN), in view of their distinct role in initiation, spread and synchronization of SWDs (Depaulis et al. 2016; Lüttjohann and van Luijtelaar 2015; Crunelli et al. 2020). Results were iteratively analyzed, in that all possible combinations of the 2-3 simultaneous recording sites were compared by using the algorithm of Maksimenko et al., (2017). Moreover, a thorough comparison of wavelet spectra corresponding to true and false positive detections was performed and a random forest machine learning algorithm was trained to further differentiate between true and false positives. Algorithm performance was evaluated according to the guidelines of good scientific practice (Mormann et al. 2007; Kuhlmann et al. 2018) (long lasting, non-selected, pseudo-prospective 24 hours recordings with both in-sample and out-of-sample periods, evaluation against chance level prediction using surrogate statistics), and it was found to reduce the false alarm rate by on average $71.4 \%$ 
4.1 Highest SWD prediction performance is achieved with analysis of LFP signals in the close proximity of the seizure initiation network in S1

Comparison of a total of 85 combinations of recording sites within the cortico-thalamic system (Table I), revealed that prediction performance was best when based on analysis of the wavelet energy of recordings obtained by three recording electrodes within the deep layers of the somatosensory cortex. SWDs are well known to be generated in the corticothalamic system. While the exact interactions between cortex and thalamus are still a matter of debate, accumulating evidence indicates that SWDs originate from a local intracortical initiation network in the peri-oral region of the somatosensory cortex (Crunelli et al. 2020; Jarre et al. 2017; Meeren et al. 2002). In GAERS, the crucial role of layer V and VI of S1 has been highlighted, as theses layers were found to contain abnormally (i.e. hyperactively) discharging neurons, which drove neuronal activity in other cortical layers as well as thalamic activity (Lüttjohann and Pape 2019; Polack et al. 2007). These epileptogenic neurons display activity patterns strikingly similar to the precursor oscillations detected by the algorithm in the present study, including an increase in activity within up to two seconds before SWD onset and a firing frequency of around $10 \mathrm{~Hz}$ (Polack et al. 2007). Highest sensitivity of prediction was achieved by the Maksimenko et al (2017) algorithm based on analysis of wavelet energy in the deep layers of S1 (IV, V, VI), which significantly outreached all other cortico-thalamic- and intrathalamic combinations of recording sites (Figure 2A). Moreover, further classification of true and false positive detections by a trained random forest also reached highest, above chance balanced accuracies for spectra derived in the deep layers of $\mathrm{S} 1$, while classification based on intrathalamic-spectra failed to achieve above chance balanced accuracies (Figure 4B). These data are in line with the concept of a local intracortical initiation network in S1 (Meeren et al. 2002; Polack et al. 2007). Interestingly, prediction performance of the Maksimenko et al. (2017) algorithm significantly dropped upon reducing the number of simultaneous recordings sites in the deep somatosensory layers from three to two (Figure 2), further demonstrating the importance of local intracortical synchronization in S1 for SWD generation. The concurrent increase in the false alarm rate might indicate a lack of information concerning the generation of other synchronized oscillations, which might be transmitted to the deep cortical layers by other subcortical structures (Sitnikova et al. 2009; Depauls et al. 1990).

The sensitivity of SWD prediction based on three simultaneous recordings in S1 also outreached the one achieved in deep layers of M2. In view of long-range intracortical connections between S1 and M2, specifically from layer V/VI of S1 to layer V of M2 (Condé et al. 1995; Zhang and Deschênes 1997; Zakiewicz et al. 2014; Reep and Corwin 1999), the high SWD prediction performance in S1 compared to M2 suggests that SWD precursor 
activity is a locally restricted cortical phenomenon, at least with regard to the initiation zone in S1.

Prediction performance of the Maksimenko et al. (2017)-algorithm was found to differ between the two genetic model strains, in that prediction performance was generally more accurate and spectra corresponding to true and false positive detections were more differentiated in GAERS compared to WAG/Rij rats. Differences between the two models and even between different colonies of the same strain have been described for distinct electrographic features of the SWDs (Akman et al. 2010; Powell et al. 2014). It is likely that

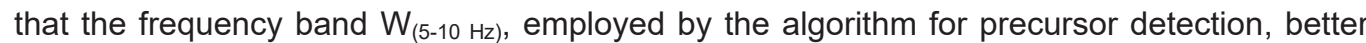
suits detection of 5-9 Hz oscillations, which have been described to preceded SWDs in GAERS (Pinault et al. 2001). In WAG/Rij rats, on the other hand, precursor activity has been described in both theta and delta frequency bands (van Luijtelaar et al. 2016; van Luijtelaar et al. 2011), implying that improved SWD prediction performance in WAG/Rij rats might require additional fine-tuning of the frequency band width applied by the Maksimenko et al. 2017 algorithm.

\subsection{Random forest machine learning algorithm for the reduction of false alarms.} Irrespective of the combination of recording sites, false alarm rates remained at a relatively high level. However, statistical comparison between wavelet spectra of true positive and false positive predictions were revealed to significantly differ in their wavelet energies in both strains and a random forest machine learning algorithm could be trained to detect such preexisting spectral differences to further differentiate between true and false positive predictions. In long lasting, out-of sample, 24 hours recordings in the deep layers of S1 in nine GAERS, which cover the full diurnal variation reported for SWD occurrence (Smyk and van Luijtelaar 2020), this additional classification of a trained random forest reduced the false alarm rate for SWD prediction by an average of $71,4 \%$

Of note, the balanced accuracy of classification depended on the approach of training (i.e. oversampling vs. undersampling) introduced to the random forest. Machine learning algorithms require a balanced training set in order for unbiased assessments of error rates to be achieved (Khan et al. 2018). With respect to SWDs, precursor and true positive predictions are an underrepresented class compared to the much larger group of interictal and false positive predictions. For balance training, random undersampling and (moderate) random oversampling (Chawla et al. 2002; Kubat et al. 1997) were used, and classification performance of two differentially trained random forests were compared. Significantly higher balanced accuracies were found for the random forest trained in the moderate oversampling approach as compared to the under-sampling approach, suggesting that undersampling 
does not include the full spectrum of variance among different types of false positive detections.

Another common source of error in machine learning algorithms is the choice of the dataset on which algorithm performance is evaluated. As mentioned above, in line with guidelines of good scientific practice (Kuhlmann et al. 2018; Mormann et al. 2007), algorithm performance was evaluated both in unseen in-sample recordings of the same rats $(30 \%$ of unseen data) as well as in lasting, non-selected, pseudo-prospective 24 hours recordings acquired in a separate group of GAERS (out-of sample evaluation). The importance of such an additional validation step can readily be inferred from the drop in algorithm performance between in-sample and out-of sample testing. Furthermore, our attempt to confront the algorithm with the full range of diurnal variations necessitated these 24 hours recordings.

Unfortunately, classification by the random forest also went along, to some degree, with a decrease in prediction sensitivity, in that 200 out of 409 SWD were correctly predicted (corresponding to a decrease in sensitivity by $41 \%$ ). The prediction of SWDs thus lacks behind the performance of prediction systems aimed at focal convulsive seizures, reaching sensitivities of prediction up to around 90\% (Kiral-Kornek et al. 2018; Khan et al. 2018; Kuhlmann et al. 2018). Of note, SWDs in absence epilepsy constitute a type of seizure that is fundamental different from focal convulsive seizures, in terms of pharmacological profile, frequency of occurrence, pathomechansms, and interictal spike patterns (Depaulis and van Luijtelaar 2006). Moreover, the moderate performance of SWD prediction may relate to interindividual differences, which are visible in both in-sample and out-of sample validation. Spatial variance between the position of the recording electrodes relative to the initiation zone in S1, or neurobiological differences in the cortical initiation network between individuals (Meeren et al. 2002) may explain these findings. As a corollary, individualized training of the random forest on long-term data obtained from a single individual may finetune and improve random forest approaches to SWD prediction.

\subsection{Possible translation to prediction of SWDs in human absence epilepsy}

SWD prediction performance of the Maksimenko et al (2017) and combined classification performance of the random forest was best for intracortical recordings obtained in close proximity to the seizure initiation network in S1. These findings provide an interesting perspective for SWD prediction in humans using surface EEG recordings. As in the genetic rat models, a local cortical initiation site of SWDs has been identified using EEG and MEG recordings combined with non-linear association analysis in children with absence epilepsy 
(Westmijse et al. 2009; Ossenblok et al. 2019). Moreover, Gupta and colleagues (2011) identified pre-ictal sources of activity, occurring approximately 1 second prior to SWDs. Of note, the exact location of the cortical SWD onset zone is variable between individual children and pre-ictal activity was reported to be most pronounced in the delta frequency range. Fine tuning of the frequency bands analyzed by the Maksimenko et al algorithm, and training of the random forest on long-lasting EEG recordings in an individual child, are thus promising possibilities paving the way for SWD prediction in children.

Wavelet analysis is a fast and reliable method for assessing non-stationary signals like LFPor EEG recordings (Hramov et al. 2015). Together with the fast temporal precision of EEG and LFP recordings, this approach allows a detection of fast and short lasting events like SWD precursors and opens the door for an implementation in an on-line setting aimed at real time prediction and prevention (Maksimenko et al. 2017) with as little interference to the overall brain activity as possible (van Luijtelaar et al. 2017; Osterhagen et al. 2010). Such a treatment approach might go along with a strong relief of side-effects often reported for the commonly used chronic pharmaceutical interventions (Crunelli et al. 2020).

\section{References}

2016 Community Survey of Epilepsy Innovation Institute (Ei2) (2016). USA. Available online at https://www.epilepsy.com/sites/core/files/atoms/files/community-survey-report2016\%20V2.pdf.

Akman, Ozlem; Demiralp, Tamer; Ates, Nurbay; Onat, Filiz Yilmaz (2010): Electroencephalographic differences between WAG/Rij and GAERS rat models of absence epilepsy. In Epilepsy research 89 (2), pp. 185-193. DOI: 10.1016/j.eplepsyres.2009.12.005.

Birjandtalab, Javad; Baran Pouyan, Maziyar; Cogan, Diana; Nourani, Mehrdad; Harvey, Jay (2017): Automated seizure detection using limited-channel EEG and non-linear dimension reduction. In Computers in Biology and Medicine 82, pp.49-58. DOI: 10.1016/j.compbiomed.2017.01.011.

Chawla, N. V.; Bowyer, K. W.; Hall, L. O.; Kegelmeyer, W. P. (2002): SMOTE: Synthetic Minority Over-sampling Technique. In jair 16, pp. 321-357. DOI: 10.1613/jair.953.

Condé, F.; Maire-Lepoivre, E.; Audinat, E.; Crépel, F. (1995): Afferent connections of the medial frontal cortex of the rat. II. Cortical and subcortical afferents. In The Journal of comparative neurology 352 (4), pp. 567-593. DOI: 10.1002/cne.903520407.

Crunelli, Vincenzo; Lőrincz, Magor L.; McCafferty, Cian; Lambert, Régis C.; Leresche, Nathalie; Di Giovanni, Giuseppe; David, François (2020): Clinical and experimental insight into pathophysiology, comorbidity and therapy of absence seizures. In Brain 143 (8), pp. 2341-2368. DOI: 10.1093/brain/awaa072. 
Daily, Mike; Medasani, Swarup; Behringer, Reinhold; Trivedi, Mohan (2017): Self-Driving Cars. In Computer 50 (12), pp. 18-23. DOI: 10.1109/MC.2017.4451204.

Depaulis, Antoine; Charpier, Stéphane (2018): Pathophysiology of absence epilepsy: Insights from genetic models. In Neuroscience letters 667, pp. 53-65. DOI: 10.1016/j.neulet.2017.02.035.

Depaulis, Antoine; David, Olivier; Charpier, Stéphane (2016): The genetic absence epilepsy rat from Strasbourg as a model to decipher the neuronal and network mechanisms of generalized idiopathic epilepsies. In Journal of neuroscience methods 260, pp. 159-174. DOI: 10.1016/j.jneumeth.2015.05.022.

Depaulis, Antoine; van Luijtelaar, Gilles (2006): Genetic models of absence epilepsy. In Pitkanen, A., Schwartzkroin, P.P., Moshe, S.L. (Ed.): Models of Seizures and Epilepsy. SanDiego: Elsevier Academic Press.

Depauls, A.; Vergnes, M.; Liu, Z.; Kempf, E.; Marescaux, C. (1990): Involvement of the nigral output pathways in the inhibitory control of the substantia nigra over generalized nonconvulsive seizures in the rat. In Neuroscience 39 (2), pp. 339-349. DOI: 10.1016/03064522(90)90272-6.

Eberlein, Matthias; Müller, Jens; Yang, Hongliu; Walz, Simon; Schreiber, Janina; Tetzlaff, Ronald et al. (2019): Evaluation of machine learning methods for seizure prediction in epilepsy. In Current Directions in Biomedical Engineering 5 (1), pp. 109-112. DOI: 10.1515/cdbme-2019-0028.

Gupta, Disha; Ossenblok, Pauly; van Luijtelaar, Gilles (2011): Space-time network connectivity and cortical activations preceding spike wave discharges in human absence epilepsy: a MEG study. In Medical \& biological engineering \& computing 49 (5), pp. 555-565. DOI: $10.1007 / \mathrm{s} 11517-011-0778-3$.

Hramov, Alexander E.; Koronovskii, Alexey A.; Makarov, Valeri A.; Pavlov, Alexey N.; Sitnikova, Evgenia (2015): Wavelets in Neuroscience. Heidelberg: Springer (Springer Series in Synergetics). Available online at http://gbv.eblib.com/patron/FullRecord.aspx?p=1802957.

Jarre, Guillaume; Altwegg-Boussac, Tristan; Williams, Mark S.; Studer, Florian; Chipaux, Mathilde; David, Olivier et al. (2017): Building Up Absence Seizures in the Somatosensory Cortex: From Network to Cellular Epileptogenic Processes. In Cerebral cortex (New York, N.Y. : 1991) 27 (9), pp. 4607-4623. DOI: 10.1093/cercor/bhx174.

Khan, Haidar; Marcuse, Lara; Fields, Madeline; Swann, Kalina; Yener, Bulent (2018): Focal Onset Seizure Prediction Using Convolutional Networks. In IEEE transactions on bio-medical engineering 65 (9), pp. 2109-2118. DOI: 10.1109/TBME.2017.2785401.

Kiral-Kornek, Isabell; Roy, Subhrajit; Nurse, Ewan; Mashford, Benjamin; Karoly, Philippa; Carroll, Thomas et al. (2018): Epileptic Seizure Prediction Using Big Data and Deep Learning: Toward a Mobile System. In EBioMedicine 27, pp. 103-111. DOI: 10.1016/j.ebiom.2017.11.032.

Kubat, Miroslav; Holte, Robert; Matwin, Stan (1997): Learning when negative examples abound. In Maarten Someren, Gerhard Widmer (Eds.): Machine Learning: ECML-97. 9th 
European Conference on Machine Learning Prague, Czech Republic, April 23-25, 1997 Proceedings, vol. 1224. Berlin, Heidelberg: Springer (Lecture Notes in Computer Science, Lecture Notes in Artificial Intelligence, 1224), pp. 146-153.

Kuhlmann, Levin; Lehnertz, Klaus; Richardson, Mark P.; Schelter, Björn; Zaveri, Hitten P. (2018): Seizure prediction - ready for a new era. In Nature reviews. Neurology 14 (10), pp. 618-630. DOI: 10.1038/s41582-018-0055-2.

Lopes Da Silva; Blanes; Kalitzin; Parra; Suffczynski; Velis (2003): Epilepsies as Dynamical Diseases of Brain Systems: Basic Models of the Transition Between Normal and Epileptic Activity. In Epilepsia 44, pp. 72-83. DOI: 10.1111/j.0013-9580.2003.12005.x.

Lüttjohann, Annika; Pape, Hans-Christian (2019): Regional specificity of cortico-thalamic coupling strength and directionality during waxing and waning of spike and wave discharges. In Scientific reports 9 (1), p. 2100. DOI: 10.1038/s41598-018-37985-7.

Lüttjohann, Annika; van Luijtelaar, Gilles (2012): The dynamics of cortico-thalamo-cortical interactions at the transition from pre-ictal to ictal LFPs in absence epilepsy. In Neurobiology of disease 47 (1), pp. 49-60. DOI: 10.1016/j.nbd.2012.03.023.

Lüttjohann, Annika; van Luijtelaar, Gilles (2015): Dynamics of networks during absence seizure's on- and offset in rodents and man. In Front. Physiol. 6, p. 16. DOI: 10.3389/fphys.2015.00016.

Maksimenko, Vladimir A.; van Heukelum, Sabrina; Makarov, Vladimir V.; Kelderhuis, Janita; Lüttjohann, Annika; Koronovskii, Alexey A. et al. (2017): Absence Seizure Control by a Brain Computer Interface. In Scientific reports 7 (1), p. 2487. DOI: 10.1038/s41598-017-02626-y.

Meeren, Hanneke K. M.; Pijn, Jan Pieter M.; van Luijtelaar, Egidius L. J. M.; Coenen, Anton M. L.; Lopes da Silva, Fernando H. (2002): Cortical Focus Drives Widespread Corticothalamic Networks during Spontaneous Absence Seizures in Rats. In J. Neurosci. 22 (4), pp. 1480-1495. DOI: 10.1523/JNEUROSCI.22-04-01480.2002.

Mormann, Florian; Andrzejak, Ralph G.; Elger, Christian E.; Lehnertz, Klaus (2007): Seizure prediction: the long and winding road. In Brain 130 (2), pp. 314-333. DOI: 10.1093/brain/awl241.

Ossenblok, Pauly; van Houdt, Petra; Colon, Albert; Stroink, Hans; van Luijtelaar, Gilles (2019): A network approach to investigate the bi-hemispheric synchrony in absence epilepsy. In Clinical neurophysiology : official journal of the International Federation of Clinical Neurophysiology 130 (9), pp. 1611-1619. DOI: 10.1016/j.clinph.2019.05.034.

Osterhagen, Lasse; Breteler, Marinus; van Luijtelaar, Gilles (2010): Does arousal interfere with operant conditioning of spike-wave discharges in genetic epileptic rats? In Epilepsy research 90 (1), pp. 75-82. DOI: 10.1016/j.eplepsyres.2010.03.010.

Panayiotopoulos, C. P.; Chroni, E.; Daskalopoulos, C.; Baker, A.; Rowlinson, S.; Walsh, P. (1992): Typical absence seizures in adults: clinical, EEG, video-EEG findings and diagnostic/syndromic considerations. In Journal of neurology, neurosurgery, and psychiatry 55 (11), pp. 1002-1008. DOI: 10.1136/jnnp.55.11.1002. 
Paxinos, G. Watson C. (1998): The Rat Brain in Stereotactic Coordinates. London: Academic Press, Ltd.

Pinault, D.; Vergnes, M.; Marescaux, C. (2001): Medium-voltage 5-9-Hz oscillations give rise to spike-and-wave discharges in a genetic model of absence epilepsy: in vivo dual extracellular recording of thalamic relay and reticular neurons. In Neuroscience 105 (1), pp. 181-201. DOI: 10.1016/s0306-4522(01)00182-8.

Polack, Pierre-Olivier; Guillemain, Isabelle; Hu, Emilie; Deransart, Colin; Depaulis, Antoine; Charpier, Stéphane (2007): Deep layer somatosensory cortical neurons initiate spike-andwave discharges in a genetic model of absence seizures. In The Journal of neuroscience : the official journal of the Society for Neuroscience 27 (24), pp.6590-6599. DOI: 10.1523/JNEUROSCI.0753-07.2007.

Powell, Kim L.; Tang, Howard; Ng, Caroline; Guillemain, Isabelle; Dieuset, Gabriel; Dezsi, Gabi et al. (2014): Seizure expression, behavior, and brain morphology differences in colonies of Genetic Absence Epilepsy Rats from Strasbourg. In Epilepsia 55 (12), pp. 19591968. DOI: 10.1111/epi.12840.

Ratner, Mark (2015): IBM's Watson Group signs up genomics partners. In Nature biotechnology 33 (1), pp. 10-11. DOI: 10.1038/nbt0115-10.

Reep, R. L.; Corwin, J. V. (1999): Topographic organization of the striatal and thalamic connections of rat medial agranular cortex. In Brain research 841 (1-2), pp. 43-52. DOI: 10.1016/s0006-8993(99)01779-5.

Seizure Gauge Challenge (2017). Edited by Epilepsy Foundation. USA. Available online at https://www.epilepsy.com/about-us/research-and-new-therapies/innovation/epilepsyinnovation-institute/seizure-gauge-challenge.

Sitnikova, Evgenia; Hramov, Alexander E.; Koronovsky, Alexey A.; van Luijtelaar, Gilles (2009): Sleep spindles and spike-wave discharges in EEG: Their generic features, similarities and distinctions disclosed with Fourier transform and continuous wavelet analysis. In Journal of neuroscience methods 180 (2), pp. 304-316. DOI: 10.1016/j.jneumeth.2009.04.006.

Sitnikova, Evgenia; van Luijtelaar, Gilles (2007): Electroencephalographic Characterization of Spike-Wave Discharges in Cortex and Thalamus in WAG/Rij Rats. In Epilepsia 0 (0), 070810012536001-??? DOI: 10.1111/j.1528-1167.2007.01250.x.

Smyk, Magdalena K.; van Luijtelaar, Gilles (2020): Circadian Rhythms and Epilepsy: A Suitable Case for Absence Epilepsy. In Frontiers in neurology 11, p. 245. DOI: 10.3389/fneur.2020.00245.

Sorokin, Jordan M.; Paz, Jeanne T.; Huguenard, John R. (2016): Absence seizure susceptibility correlates with pre-ictal $\beta$ oscillations. In Journal of physiology, Paris $110(4 \mathrm{Pt}$ A), pp. 372-381. DOI: 10.1016/j.jphysparis.2017.05.004.

van Luijtelaar, G.; Zobeiri, M. (2014): Progress and outlooks in a genetic absence epilepsy model (WAG/Rij). In Current medicinal chemistry 21 (6), pp. 704-721. DOI: 10.2174/0929867320666131119152913. 
van Luijtelaar, G. V.; Zobeiri, M.; Lüttjohann, A.; Depaulis, A. (2017): Experimental Treatment Options in Absence Epilepsy. In Current pharmaceutical design 23 (37). DOI: 10.2174/1381612823666171017170226.

van Luijtelaar, Gilles; Hramov, Alexander; Sitnikova, Evgenia; Koronovskii, Alexei (2011): Spike-wave discharges in WAG/Rij rats are preceded by delta and theta precursor activity in cortex and thalamus. In Clinical neurophysiology : official journal of the International Federation of Clinical Neurophysiology 122 (4), pp. 687-695. DOI: 10.1016/j.clinph.2010.10.038.

van Luijtelaar, Gilles; Lüttjohann, Annika; Makarov, Vladimir V.; Maksimenko, Vladimir A.; Koronovskii, Alexei A.; Hramov, Alexander E. (2016): Methods of automated absence seizure detection, interference by stimulation, and possibilities for prediction in genetic absence models. In Journal of neuroscience methods 260, pp. 144-158. DOI: 10.1016/j.jneumeth.2015.07.010.

Walter, Martin; Alizadeh, Sarah; Jamalabadi, Hamidreza; Lueken, Ulrike; Dannlowski, Udo; Walter, Henrik et al. (2019): Translational machine learning for psychiatric neuroimaging. In Progress in neuro-psychopharmacology \& biological psychiatry 91, pp. 113-121. DOI: 10.1016/j.pnpbp.2018.09.014.

Westmijse, Inge; Ossenblok, Pauly; Gunning, Boudewijn; van Luijtelaar, Gilles (2009): Onset and propagation of spike and slow wave discharges in human absence epilepsy: A MEG study. In Epilepsia 50 (12), pp. 2538-2548. DOI: 10.1111/j.1528-1167.2009.02162.x.

Zakiewicz, Izabela M.; Bjaalie, Jan G.; Leergaard, Trygve B. (2014): Brain-wide map of efferent projections from rat barrel cortex. In Frontiers in neuroinformatics 8, p. 5. DOI: 10.3389/fninf.2014.00005.

Zhang, Z. W.; Deschênes, M. (1997): Intracortical axonal projections of lamina VI cells of the primary somatosensory cortex in the rat: a single-cell labeling study. In The Journal of neuroscience : the official journal of the Society for Neuroscience 17 (16), pp. 6365-6379.

\section{2}


Figure legends and tables

856

Figure 1: Exemplary local field potential recordings in the deep somatosensory cortex of a GAERS (right) as well as simultaneously recorded LFPs the deep somatosensory cortex and vertral-postero-medial thalamic nucleus (VPM) of a WAG/Rij rat (upper left panel and lower left panel, respectively). Arrows indicates the onset of the SWD, determined according to the criteria outlined by van Luijtelaar \& Coenen (1986), taking the peak of the first spike of twice the background as reference for SWD onset.

Figure 2: Wavelet analysis for SWD prediction. Relative sensitivity (A) and average false alarm rate $(C)$ of SWD prediction for different combinations of recording sites in the corticothalamic system, obtained by the Maksimenko et al. (2017) algorithm. LFPs, simultaneously recorded in the cortico-thalamic system of WAG-Rij rats (see methods), were analyzed in combinations of either two or three recording sites. Results from all 85 combinations are presented in Table I. To avoid type II errors, all combinations of recording sites were grouped as either 'CC' (two intracortical recording sites in S1), 'CT' (one cortical recording site in S1 and one thalamic recording site), 'TT' (two intrathalamic recording sites, 'CCC' (three intracortical recording sites in S1), 'CCT' (two cortical recording sites in S1 and one thalamic recording site), 'CTT' (one cortical recording site in $\mathrm{S} 1$ and two thalamic recording sites), 'TTT' (three intrathalamic recording sites) or 'MCCC' (three intracortical recording sites in the secondary motor cortex), respectively. B, C: Results of post-hoc comparison verified by ANOVA, with ${ }^{* * *}$ indicating significance at a $p<0.001$ level for sensitivity of prediction (B) and false alarm rate (D), respectively. E: Relationship of false alarm rates and average sensitivity of SWD prediction for different combinations of recording sites in the cortico-thalamic system of WAG/Rij rats, analyzed by the Maksimenko et al. (2017) algorithm. Note highest sensitivity with a low false alarm rate for prediction based on three intracortical recordings in S1 (blue triangle) that outperforms all other combinations of recording sites. Further note the negative correlation between both indicators of SWD prediction performance $(r=-0.716 ; p<0.001)$, indicating that higher SWD prediction sensitivity at any given combination of recording sites does not occur at the trade-off of a high false alarm rate. 
Figure 3: SWD prediction in two genetic rat models of absence epilepsy. A, B: Average sensitivity of SWD prediction (A) and false alarm rate expressed in number false positives per hour (nFP/h) (B) achieved by the Maksimenko et al. (2017) algorithm assessed in 4 hours lasting LFP recordings, obtained in layers IV, V and VI of S1 in GAERS and WAG/Rij rats.

C - E: Comparison of wavelet spectra of true and false positive predictions. An exemplary LFP trace depicting a pre-SWD -> SWD transition is presented in (C). Onset of SWD is marked by red vertical line termed 2. The corresponding spectrogram of a true positive detection identified in intracortical LFP recordings in S1 of a GAERS is shown in (D). Time point -0.5 to 0 (red rectangle termed 1 ) features the analysis window (window size $500 \mathrm{~ms}$ ) in which the true positive precursor is detected. An exemplary spectrogram of a false positive detection is shown in (E). Again, time point -0.5 to 0 features the analysis window (window size $500 \mathrm{~ms}$ ) in which the false positive precursor is detected. F: Statistical comparison of the product of wavelet energy, assessed in the frequency bands $W_{(5-10 \mathrm{~Hz})}, \mathrm{W}_{(3-5 \mathrm{~Hz})}$ and $\mathrm{W}_{(7-20 \mathrm{~Hz})}$ (Maksimenko et al. 2017), between true and false positives in WAG/Rij rats. E: Statistical comparison of the product of wavelet energy, assessed in the frequency bands $W_{(5-10 \mathrm{~Hz})}, \mathrm{W}_{(3-}$ $5 \mathrm{~Hz})$ and $W_{(7-20 \mathrm{~Hz})}$ (Maksimenko et al. 2017), between true and false positives in GAERS. ${ }^{* * *}$ indicates a significant difference verified by ANOVA at level of $p<0.001 ;{ }^{* *}$ at a level of $p<0.01$; and * at level of $p<0.05$.

Figure 4: Differentiation between true- and false positives by a random forest machine learning algorithm. A: Schematic representation of the random forest machine learning algorithm for differentiation between true positive and false positive predictions. After wavelet analysis of either two or three simultaneously recorded LFP traces, the wavelet energies $\left(\mathrm{W}_{(5-10 \mathrm{~Hz})}, \mathrm{W}_{(3-5 \mathrm{~Hz})}\right.$ and $\left.\mathrm{W}_{(7-20 \mathrm{~Hz})}\right)$ extracted in each trace are fed to a random forest composed of 1000 decision trees. Final classification of the random forest is yielded from a majority voting of the different trees (see methods for detail). B: Out-of-sample performance (expressed as balanced accuracy) of random forests. Training in an undersampling approach on wavelet spectra derived from recordings in layers $V$ and $V I$ of $S 1$ (CC), recordings in layers IV, $\mathrm{V}$ and $\mathrm{VI}$ of $\mathrm{S} 1$ (CCC), recordings in layers IV, $\mathrm{VI}$ of $\mathrm{S} 1$ and VPM (CCT), recordings in layer VI of S1, VPM and RTN (CTT), recordings in VPM, CRTN and Po (TTT) of WAG/Rij rats at a sensitivity of $60 \%$, and recordings in layers IV, V and VI of S1 of GAERS at a sensitivity of $60 \%$ (GCCC) or $90 \%$ (GCCC $90 \%$ ). Numbers in GAERS groups $(1844,161,145)$ refer to the different amount of true/false positive fragments, with which the random forest was trained. Stars in B indicate a significant classification above chance as validated by surrogate statistics with * indicating significance at a $p<0.05,{ }^{* *} p<0.01$ and ${ }^{* * *}$ 
$921 p<0.001$ level. C: Table of achieved average balanced accuracies achieved by analysis of 922 the different combinations of recording sites. D: Statistics between group comparison of 923 balanced accuracies performed with ANOVA with * indicating significance at a $p<0.05$, ** $924 p<0.01$ and ${ }^{* * *} p<0.001$ level. E: Relation between classification accuracy and the number of 925 incorporated trees in the random forest.

Table I: Combinations of recording sites analyzed by the Maksimenko et al. algorithm and achieved average sensitivities of prediction and false alarm rates. Abbreviations: ctx4: layer 4 of somatosensory cortex, ctx5: layer 5 of somatosensory cortex, ctx6: layer 6 of somatosensory cortex, ATN: anterior thalamic nucleus, VPM: vertral-postero-medial thalamic nucleus, PO: posterior thalamic nucleus, rRTN: rostral reticular thalamic nucleus, cRTN: caudal reticular thalamic nucleus, Mctx5a: layer $5 a$ of secondary motor cortex, Mctx $5 b$ : layer $5 b$ of secondary motor cortex, Mctx 6 : layer 6 of secondary motor cortex

\begin{tabular}{|c|c|c|c|c|c|c|c|}
\hline $\begin{array}{l}\text { number of } \\
\text { simultaneous } \\
\text { recording } \\
\text { sites }\end{array}$ & $\begin{array}{c}\text { combination } \\
\text { number }\end{array}$ & area 1 & area 1 & area 3 & $\begin{array}{l}\text { abbreviation } \\
\text { in text and } \\
\text { figures }\end{array}$ & $\begin{array}{c}\text { average } \\
\text { sensitivity }\end{array}$ & $\begin{array}{c}\text { average } \\
\mathrm{nFP} / \mathrm{h}\end{array}$ \\
\hline \multirow[t]{20}{*}{3} & 1 & ctx 4 & ctx 5 & $\operatorname{ctx} 6$ & $\mathrm{CCC}$ & 61,755 & 85,962 \\
\hline & 2 & ctx 4 & $\operatorname{ctx} 5$ & Po & СCT & 48,392 & 65,199 \\
\hline & 3 & $\operatorname{ctx} 4$ & $\operatorname{ctx} 5$ & ATN & ССТ & 45,974 & 85,363 \\
\hline & 4 & $\operatorname{ctx} 4$ & $\operatorname{ctx} 5$ & rRTN & ССТ & 44,230 & 69,947 \\
\hline & 5 & ctx 4 & ctx 5 & cRTN & CCT & 50,600 & 58,431 \\
\hline & 6 & $\operatorname{ctx} 4$ & $\operatorname{ctx} 5$ & VPM & ССТ & 46,007 & 61,826 \\
\hline & 7 & $\operatorname{ctx} 4$ & $\operatorname{ctx} 6$ & Po & CCT & 50,718 & 68,470 \\
\hline & 8 & $\operatorname{ctx} 4$ & $\operatorname{ctx} 6$ & ATN & ССТ & 48,932 & 82,776 \\
\hline & 9 & $\operatorname{ctx} 4$ & ctx 6 & rRTN & ССТ & 45,690 & 71,436 \\
\hline & 10 & $\operatorname{ctx} 4$ & $\operatorname{ctx} 6$ & cRTN & ССТ & 51,823 & 60,125 \\
\hline & 11 & $\operatorname{ctx} 4$ & $\operatorname{ctx} 6$ & VPM & ССТ & 50,269 & 58,889 \\
\hline & 12 & $\operatorname{ctx} 5$ & $\operatorname{ctx} 6$ & Po & ССТ & 48,880 & 79,424 \\
\hline & 13 & $\operatorname{ctx} 5$ & $\operatorname{ctx} 6$ & ATN & ССТ & 48,345 & 95,587 \\
\hline & 14 & $\operatorname{ctx} 5$ & $\operatorname{ctx} 6$ & rRTN & ССТ & 51,354 & 65,995 \\
\hline & 15 & $\operatorname{ctx} 5$ & $\operatorname{ctx} 6$ & cRTN & ССТ & 48,963 & 72,081 \\
\hline & 16 & $\operatorname{ctx} 5$ & $\operatorname{ctx} 6$ & VPM & ССТ & 48,708 & 62,217 \\
\hline & 17 & $\operatorname{ctx} 4$ & Po & ATN & СTT & 36,121 & 98,180 \\
\hline & 18 & $\operatorname{ctx} 4$ & Po & rRTN & CTT & 35,171 & 97,276 \\
\hline & 19 & $\operatorname{ctx} 4$ & Po & cRTN & СTT & 35,430 & 95,410 \\
\hline & 20 & ctx 4 & Po & VPM & CTT & 34,470 & 99,526 \\
\hline
\end{tabular}




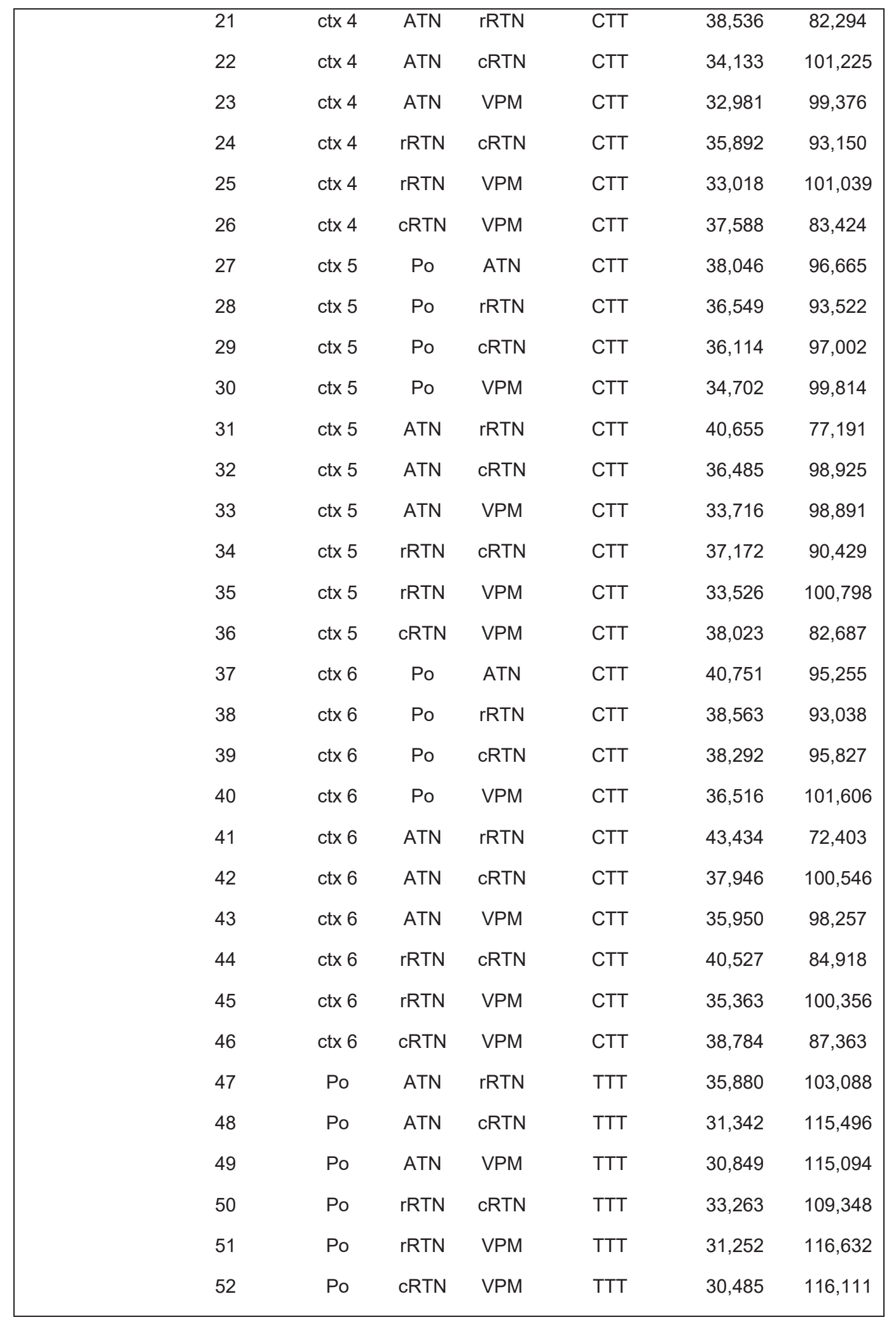




\begin{tabular}{|c|c|c|c|c|c|c|c|}
\hline & 53 & ATN & rRTN & cRTN & TTT & 36,646 & 89,893 \\
\hline & 54 & ATN & rRTN & VPM & TTT & 34,497 & 98,061 \\
\hline & 55 & ATN & cRTN & VPM & TTT & 30,137 & 110,576 \\
\hline & 56 & rRTN & cRTN & VPM & TTT & 30,907 & 115,390 \\
\hline & 57 & Mctx 5a & $\begin{array}{c}\text { Mctx } \\
5 b\end{array}$ & Mctx 6 & MCCC & 33,330 & 129,803 \\
\hline 2 & 1 & ctx 4 & $\operatorname{ctx} 5$ & & CC & 31,173 & 211,365 \\
\hline & 2 & $\operatorname{ct} x 4$ & ctx 6 & & CC & 34,619 & 209,386 \\
\hline & 3 & $\operatorname{ctx} 5$ & $\operatorname{ctx} 6$ & & CC & 33,612 & 242,989 \\
\hline & 4 & ctx 4 & VPM & & CT & 21,408 & 123,705 \\
\hline & 5 & $\operatorname{ctx} 4$ & ATN & & CT & 20,799 & 148,887 \\
\hline & 6 & $\operatorname{ctx} 4$ & Po & & CT & 21,987 & 151,854 \\
\hline & 7 & $\operatorname{ctx} 4$ & cRTN & & CT & 23,729 & 122,750 \\
\hline & 8 & $\operatorname{ctx} 4$ & rRTN & & CT & 25,276 & 130,967 \\
\hline & 9 & $\operatorname{ctx} 5$ & VPM & & CT & 23,357 & 120,332 \\
\hline & 10 & $\operatorname{ctx} 5$ & ATN & & CT & 22,728 & 158,520 \\
\hline & 11 & $\operatorname{ctx} 5$ & Po & & CT & 24,474 & 151,471 \\
\hline & 12 & $\operatorname{ctx} 5$ & cRTN & & CT & 24,874 & 130,418 \\
\hline & 13 & $\operatorname{ctx} 5$ & rRTN & & CT & 29,267 & 121,645 \\
\hline & 14 & $\operatorname{ctx} 6$ & VPM & & CT & 23,514 & 146,704 \\
\hline & 15 & ctx 6 & ATN & & CT & 24,906 & 174,084 \\
\hline & 16 & $\operatorname{ctx} 6$ & Po & & CT & 25,886 & 171,314 \\
\hline & 17 & $\operatorname{ctx} 6$ & cRTN & & CT & 25,948 & 145,599 \\
\hline & 18 & ctx 6 & rRTN & & CT & 31,349 & 137,519 \\
\hline & 19 & VPM & ATN & & TT & 10,411 & 157,414 \\
\hline & 20 & VPM & Po & & TT & 10,741 & 186,945 \\
\hline & 21 & VPM & cRTN & & TT & 14,999 & 151,043 \\
\hline & 22 & VPM & rRTN & & TT & 15,703 & 155,311 \\
\hline & 23 & ATN & Po & & TT & 12,648 & 179,928 \\
\hline & 24 & ATN & cRTN & & TT & 10,670 & 165,252 \\
\hline & 25 & ATN & rRTN & & TT & 20,339 & 142,317 \\
\hline & 26 & Po & cRTN & & TT & 11,267 & 171,176 \\
\hline
\end{tabular}




\begin{tabular}{|lcccrc|}
\hline 27 & Po & rRTN & TT & 17,575 & 166,227 \\
28 & cRTN & rRTN & TT & 21,339 & 142,157 \\
\hline
\end{tabular}

933

934

935

936

937

938

939

940

941

942

943

944

945

946

947

948

949

\begin{tabular}{|lcc|}
\hline & \multicolumn{2}{c|}{ average confusion matrix } \\
false positive & $\begin{array}{c}\text { predicted as false positive } \\
52.46 \%+-9.38\end{array}$ & $\begin{array}{c}\text { predicted as true positive } \\
47.54 \%+/-9.38\end{array}$ \\
true positive & $50.66 \%+/-8.95$ & $49.34 \%+/-8.95$ \\
& & \\
\hline & balanced accuracy & F1-score \\
rat 1 & $47,37 \%$ & $14,53 \%$ \\
rat 2 & $53,68 \%$ & $6,89 \%$ \\
rat 3 & $47,44 \%$ & $11,74 \%$ \\
rat 4 & $49,07 \%$ & $4,82 \%$ \\
rat 5 & $59,62 \%$ * & $9,14 \%$ \\
rat 6 & $51,06 \%$ & $5,44 \%$ \\
rat 7 & $51,93 \%$ & $7,18 \%$ \\
rat 8 & $50,13 \%$ & $4,25 \%$ \\
rat 9 & $47,82 \%$ & $9,68 \%$ \\
\hline
\end{tabular}
to spectra derived from 24 hours recordings in a separate group of GAERS $(n=9)$. classification as verified by surrogate statistics.

Table II: Out-of-sample performance of the random forest (trained in an undersampling approach on spectra derived from three intracortical recordings in S1 of GAERS at a sensitivity of $90 \%$ ) confronted

Depicted in the upper panel is the average confusion matrix (+/- SEM), specifying the percentage of true positives correctly classified as true positives (lower right corner), true positives incorrectly classified as false positives (lower left corner), false positives correctly classified as false positives (upper left corner) and false positives incorrectly classified as true positives (upper right corner).

Lower panel depicts the balanced accuracies and F1-scores for each individual rat. Note that the F1 score reflects the tradeoff between false alarm rate/sensitivity. Low F1 scores are reflecting the drop of sensitivity associated to the drop of false alarm rate. As our goal in this work is the latter, the low scores are justified by the high balanced accuracies. * denotes an above chance balanced accuracy of

951

952

953

954

955

956

957 
Table III: Out-of-sample performance of the random forest (trained in an oversampling approach on spectra derived from three intracortical recordings in S1 of GAERS at a sensitivity of $90 \%$ ) confronted to spectra derived from 24 hours recordings in a separate group of GAERS $(n=9)$.

Depicted in the upper panel is the average confusion matrix (+/- SEM), specifying the percentage of true positives correctly classified as true positives (lower right corner), true positives incorrectly classified as false positives (lower left corner), false positives correctly classified as false positives (upper left corner) and false positives incorrectly classified as true positives (upper right corner).

Lower panel depicts the balanced accuracies and F1-scores for each individual rat. Note that the F1 score reflects the tradeoff between false alarm rate/sensitivity. Low F1 scores are reflecting the drop of sensitivity associated to the drop of false alarm rate. As our goal in this work is the latter, the low scores are justified by the high balanced accuracies. * denotes an above chance balanced accuracy of classification as verified by surrogate statistics.

\begin{tabular}{|lcc|}
\hline & \multicolumn{2}{c|}{ average confusion matrix } \\
false positive & $\begin{array}{c}\text { predicted as false positive } \\
\text { predicted as true positive } \\
28.62 \%+/-2.56\end{array}$ \\
true positive & $46.00 \%+/-4.00$ & $54.00 \%+/-4.00$ \\
& & \\
\hline & balanced accuracy & F1-score \\
rat 1 & $70,28 \%$ * & $46,88 \%$ \\
rat 2 & $55,14 \%$ & $7,59 \%$ \\
rat 3 & $60,13 \%$ * & $16,60 \%$ \\
rat 4 & $63,98 \%$ * & $12,21 \%$ \\
rat 5 & $63,15 \%$ * & $12,02 \%$ \\
rat 6 & $59,70 \%$ * & $8,64 \%$ \\
rat 7 & $68,47 \%$ * & $13,14 \%$ \\
rat 8 & $59,00 \%$ * & $6,51 \%$ \\
rat 9 & $64,38 \%$ * & $19,71 \%$ \\
\hline
\end{tabular}

977 
GAERS

cortex (S1)
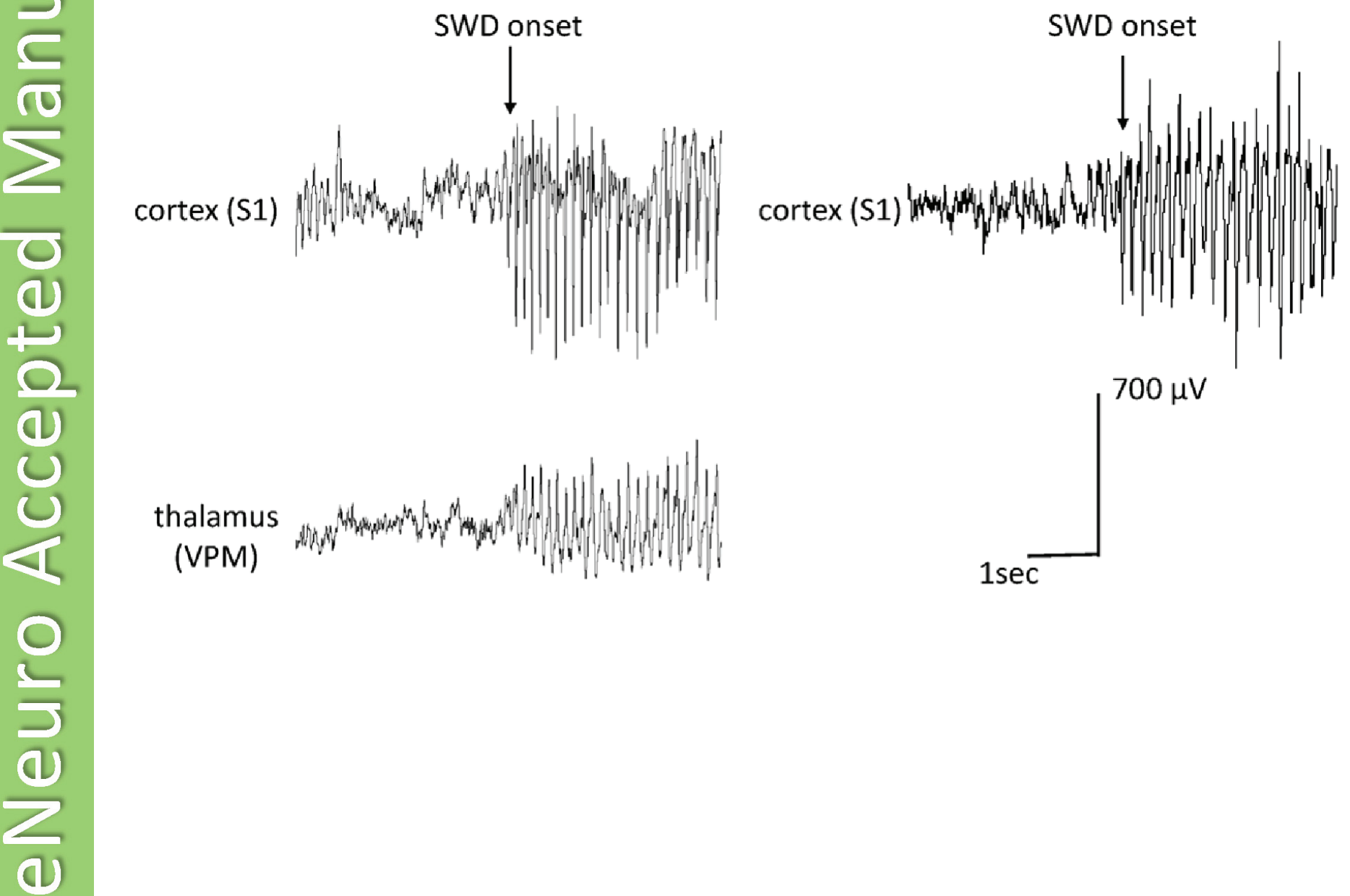

thalamus

(VPM)

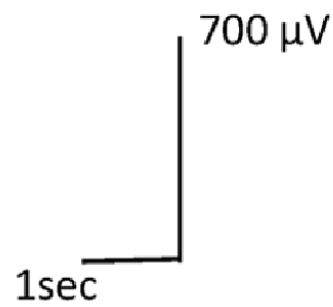



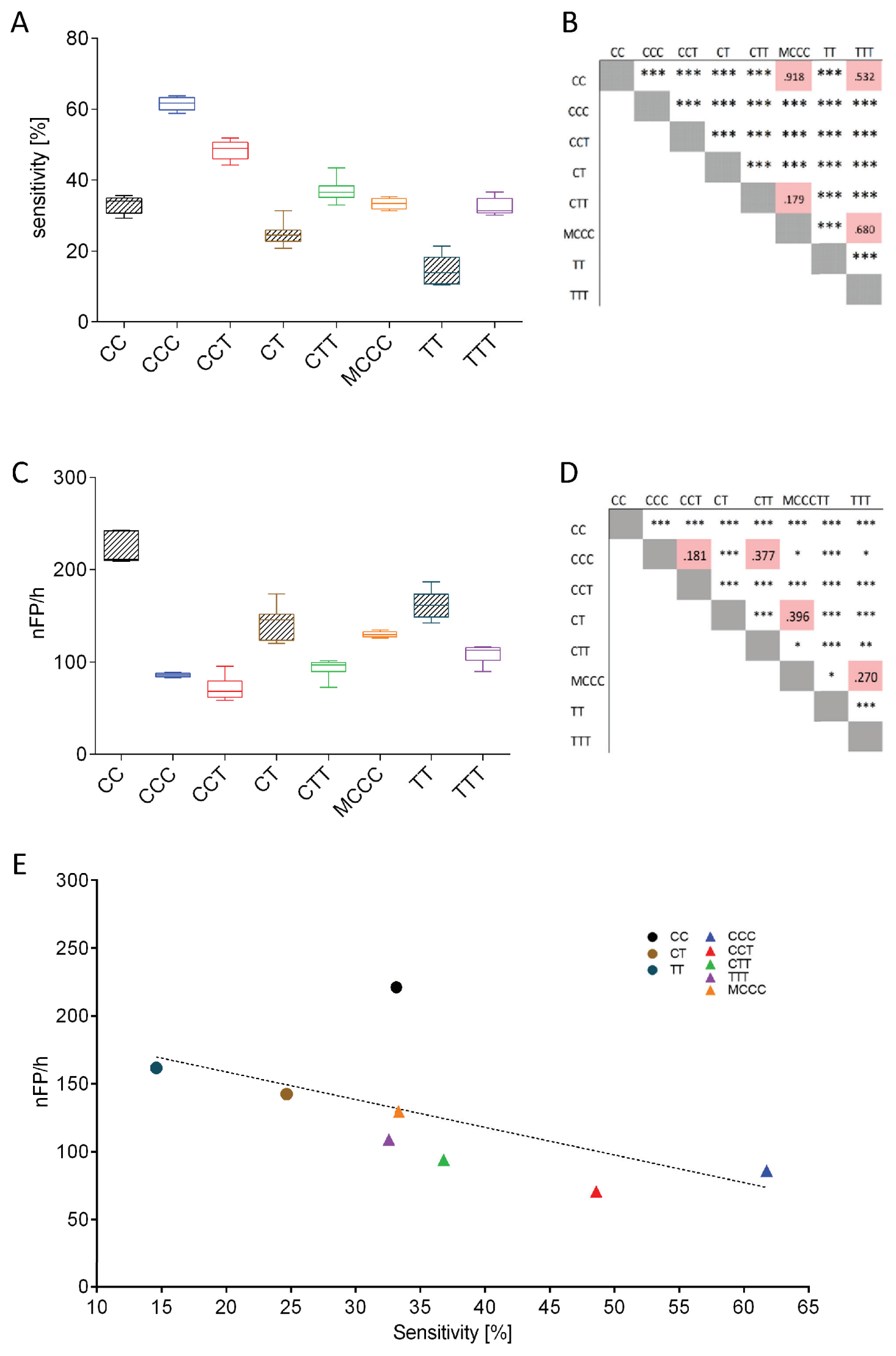
A

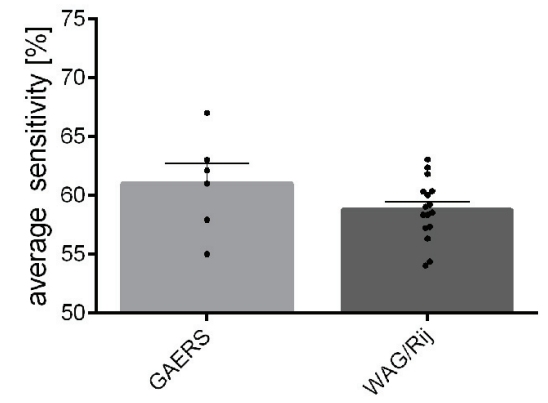

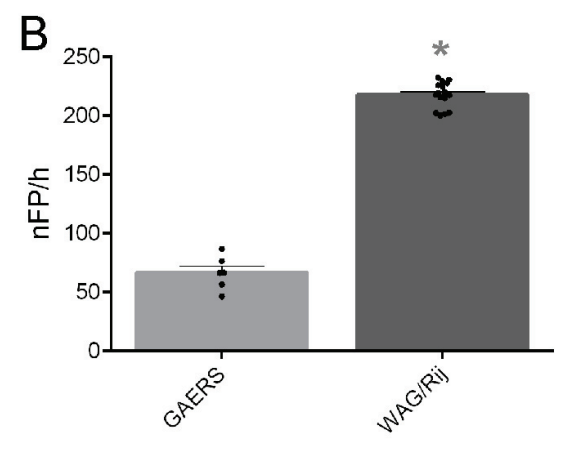

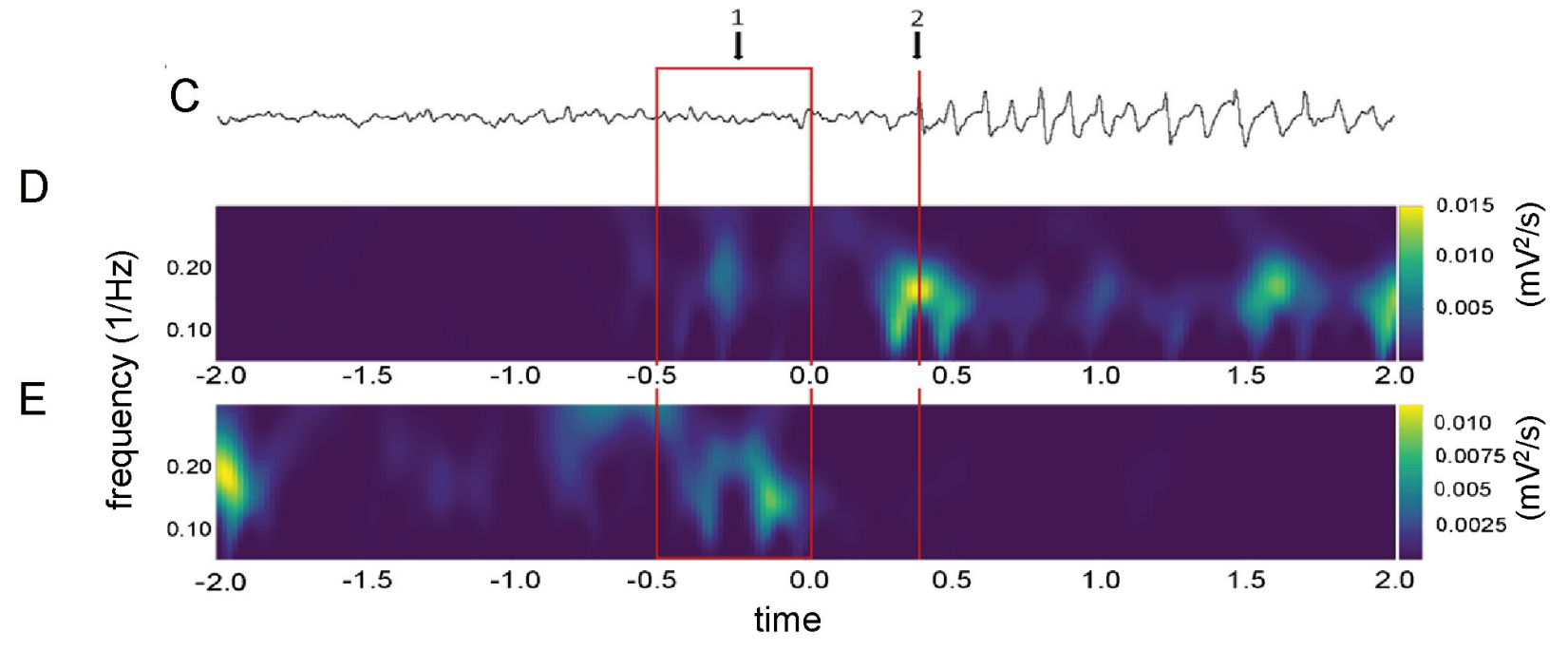

F

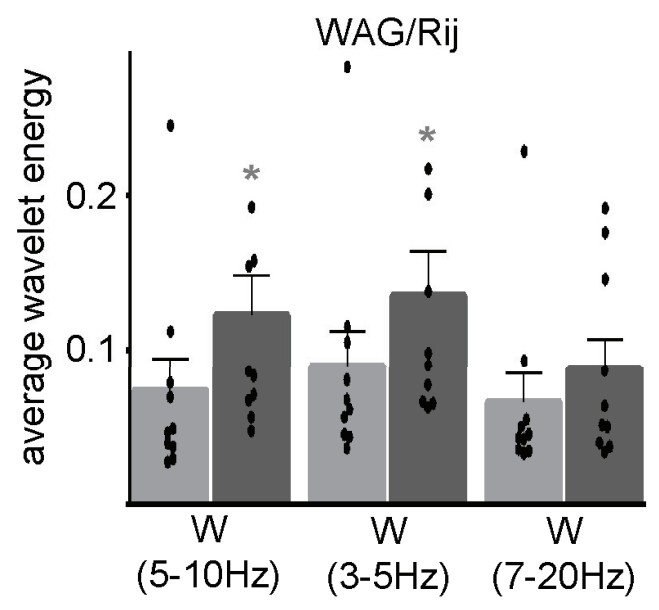

G

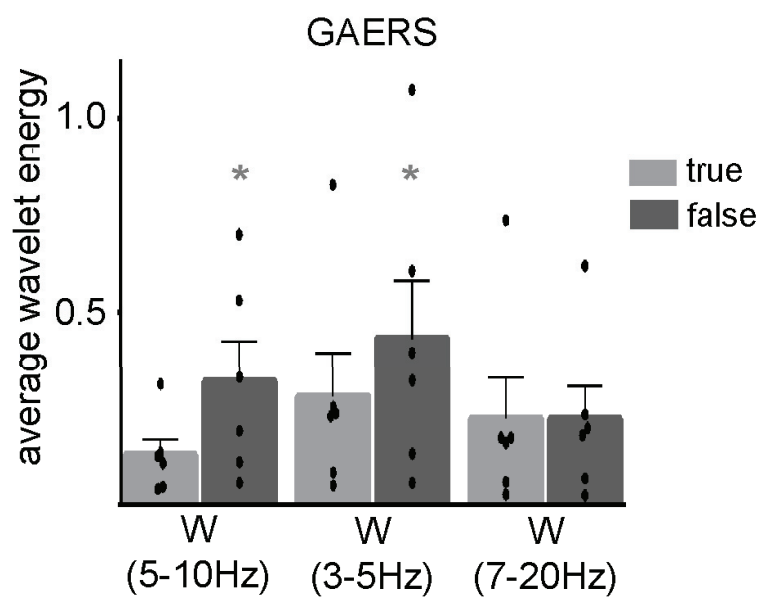


A

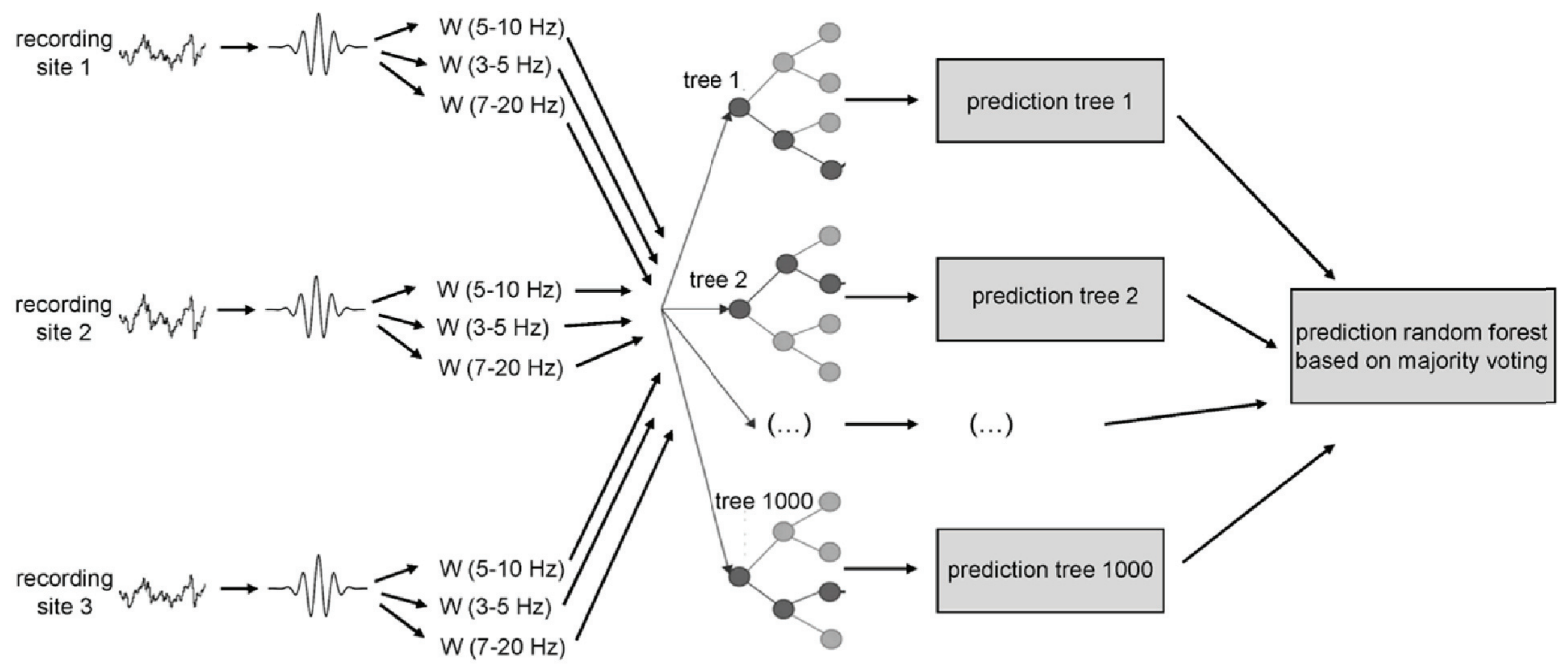

B

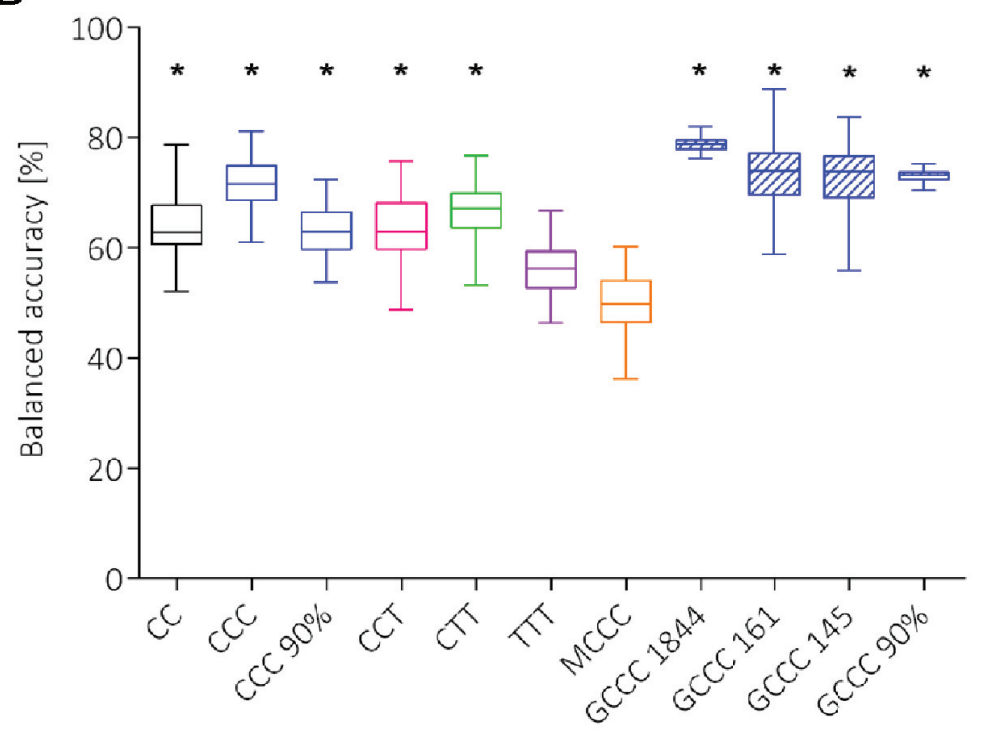

C

\begin{tabular}{|cc|}
\hline combination & $\begin{array}{c}\text { balanced } \\
\text { accuracy }\end{array}$ \\
\hline CC & $62.50 \%$ \\
CCC & $71.47 \%$ \\
CCC $90 \%$ & $63.30 \%$ \\
CCT & $66.70 \%$ \\
CTT & $63.20 \%$ \\
TTT & $56.20 \%$ \\
MCCC & $49.94 \%$ \\
GCCC 1841 & $78.80 \%$ \\
GCCC 161 & $73.58 \%$ \\
GCCC 145 & $73.00 \%$ \\
GCCC $90 \%$ & $73.10 \%$ \\
\hline
\end{tabular}

D

\begin{tabular}{|c|c|c|c|c|c|c|c|c|c|c|}
\hline & CC & 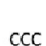 & $\begin{array}{l}\mathrm{CCC} \\
90 \%\end{array}$ & CCT & $\mathrm{CT}$ & ताT & $\mathrm{MCCC}$ & $\mathrm{GCCC}$ & GCCC & $\begin{array}{c}\mathrm{GCCC} \\
145\end{array}$ \\
\hline$C C$ & & $* * *$ & 0.398 & 0.844 & $* * * *$ & $* * *$ & $* * *$ & $* * *$ & $* * *$ & $* * *$ \\
\hline $\mathrm{CCC}$ & & & *** & *** & $* * *$ & $* * *$ & $* * *$ & ** & ** & $* * *$ \\
\hline CCC $90 \%$ & & & & 0.516 & $* * *$ & $* * * *$ & **** & **** & **** & $* * *$ \\
\hline CCT & & & & & $* * *$ & $* * *$ & *** & *** & **** & $* * *$ \\
\hline $\mathrm{CTT}$ & & & & & & $* * *$ & $m=*$ & $* * *$ & **** & $* * *$ \\
\hline TाT & & & & & & & *** & $* * *$ & $* * *$ & *** \\
\hline MeCC & & & & & & & & $* * * *$ & ***** & $* * * *$ \\
\hline GCCC1844 & & & & & & & & & **** & $* * *$ \\
\hline GCCC151 & & & & & & & & & & 0.349 \\
\hline GCCC145 & & & & & & & & & & \\
\hline
\end{tabular}

E

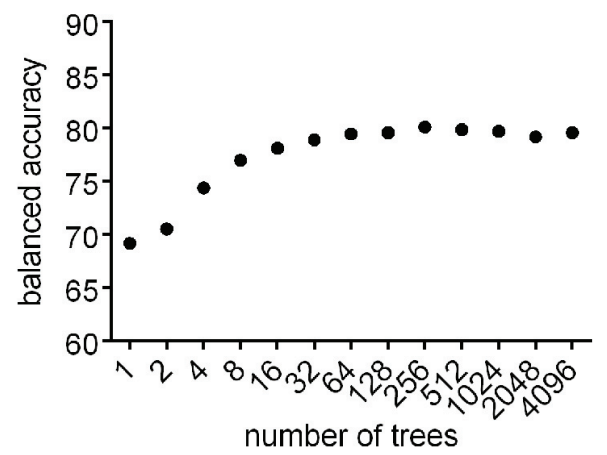

